\title{
ON THE COHOMOLOGY OF EXACT SEQUENCES OF COMPACT GROUPS
}

\author{
BY
}

SUFIAN Y. HUSSEINI

1. Introduction. If $G$ is a compact connected group, then $H^{*}(G)$, the Cech cohomology algebra with coefficients in a field $L$, is a special kind of algebra: if the characteristic of $L$ is zero, $H^{*}(G)$ is an exterior algebra; if the characteristic of $L$ is $p>0, H^{*}(G)$ is the quotient of the tensor product of an exterior algebra and a polynomial algebra. Moreover, the precise structure for $H^{*}(G)$ when $G$ is a classical Lie group has been fairly well determined $[1 ; 8]$.

Now let $K \subset G$ be a closed normal subgroup of $G$, and put $U=G / K$. Thus we have the exact sequence

$$
(\mathrm{S}): \quad 1 \rightarrow K \stackrel{i}{\rightarrow} G \stackrel{f}{\rightarrow} U \rightarrow 1
$$

of compact groups; here $i$ is the natural injection and $f$ is the natural quotient map. (S) leads to a sequence of cohomology algebras,

$$
\left(\mathrm{S}^{*}\right): \quad 1 \rightarrow H^{*}(U) \stackrel{f^{*}}{\rightarrow} H^{*}(G) \stackrel{i^{*}}{\rightarrow} H^{*}(K) \rightarrow 1,
$$

where 1 denotes the graded algebra which is zero in all positive homogeneous degrees and is isomorphic to the ground field $L$ in degree zero.

When the characteristic of the ground field $L$ is zero, then $\left(\mathrm{S}^{*}\right)$ acts as if (S) were a split sequence. (For the case of Lie groups see $[10, \S 21]$; the general case follows from it easily.) More precisely, $\left(\mathrm{S}^{*}\right)$ is an exact sequence of graded algebras, in the sense that the kernel of each homomorphism coincides with the ideal generated by the elements of positive degree in the image of the preceding homomorphism.

The purpose of this paper is to give an analysis of the structure of the sequence $\left(\mathrm{S}^{*}\right)$ when the ground field is $Z_{p}$, the prime field of characteristic $p>0$. When $K$ is connected, we show that $f^{*}$ is a monomorphism if and only if $i^{*}$ is an epimorphism, in which case $\left(\mathrm{S}^{*}\right)$ is an exact sequence of graded algebras. This is true, for example, when $H^{*}(U)$ is an exterior algebra generated by elements of odd degree. In general $\left(S^{*}\right)$ is not exact; it need not be exact, for example, when $K$ is totally disconnected. In this case we give the structure of the kernel and cokernel of $f^{*}$.

Precise statements of our main results are given in $\$ 2$. Corollaries and applications are given in $\$ 3$. For instance, we obtain a characterization of the commutativity of $G$, or the finite-dimensionality of $G$ in terms of $H^{*}(G)$.

Received by the editors May 17. 1961. 
Moreover, the class of those groups of $G$ such that $H^{*}(G)$ is an exterior algebra is shown to be closed under many operations. The proofs of the main results are given in later sections.

The author takes the opportunity to express his thanks to Professors N. E. Steenrod and J. C. Moore for their help and encouragement. This work is a part of the author's doctoral dissertation submitted to Princeton University in May, 1960.

2. The main results. The cohomology theory we use is the Cech Theory, whose coefficient domain will always be, unless otherwise specified, $Z_{p}$, the prime field of characteristic $p$.

Let $G$ be a compact connected group, and suppose that $K$ is an invariant subgroup. Consider the resulting exact sequence

$$
(\mathrm{S}): 1 \rightarrow K \stackrel{i}{\rightarrow} G \stackrel{f}{\rightarrow} U \rightarrow 1
$$

of compact groups and homomorphisms. Here $U=G / K, i$ is the natural imbedding, and $f$ is the quotient map. The study of the structure of the induced cohomology sequence,

$$
\left(\mathrm{S}^{*}\right): 1 \rightarrow B^{*}(U) \stackrel{f^{*}}{\rightarrow} H^{*}(G) \stackrel{i^{*}}{\rightarrow} B^{*}(K) \rightarrow 1,
$$

reduces in a fairly well-known manner to two cases:

(A) $K$ is totally disconnected;

(B) $K$ is connected.

Furthermore, case (A) reduces to a special case of the problem, $\left(\mathrm{A}^{\prime}\right)$ say, of the cohomological study of the locally trivial fibration of $G$ by a finite subgroup (not necessarily invariant) $\Gamma$, isomorphic to $Z_{p^{n}}$. In $(2.2)$ we state the results regarding $\left(A^{\prime}\right)$; the result of $(2.3)$ is related to $(A)$, while those of $(2.4)$ are devoted to $(\mathrm{B})$.

(2.1) Let $G$ be a compact connected group, and let $\Gamma$ be a commutative finite subgroup of $G$. Denote the space of left cosets of $G$ modulo $\Gamma$ by $U$, and let $f$ be the natural map

$$
f: G \rightarrow U
$$

Observe now that $f$ is a locally trivial fibration of $G$ by $\Gamma$. Consider next

$$
\gamma: E=E^{\infty}(\Gamma) \rightarrow B=B^{\infty}(\Gamma),
$$

the $\infty$-universal bundle of $\Gamma$ constructed by Milnor [7]. Thus there exists a mapping,

$$
\chi: U \rightarrow B,
$$

inducing $f: G \rightarrow U$. (If $G$ is a Lie group, then the usual classification theorem for fibre bundles implies the existence of $\chi$. The general case can be reduced to this special case by choosing a closed invariant subgroup, $K_{\alpha}$, such that 
$K_{\alpha} \cap \Gamma=\{1\}$ and $G / K_{\alpha}$ is a Lie group.)

Definition (2.1.1). The mapping $\chi$ inducing the fibration $f: G \rightarrow U$ is called the characteristic map of $f$; and im $\chi^{*} \subset H^{*}(U)$, the characteristic algebra of $f$, which will be denoted by $\operatorname{ch}(f)$.

Definition (2.1.2). A sequence

$$
\cdots \rightarrow A_{n-1} \rightarrow A_{n} \rightarrow A_{n+1} \rightarrow \cdots
$$

of commutative graded algebras and homomorphisms over $Z_{p}$ is said to be exact if the kernel of each homomorphism is equal to the ideal generated by the elements of positive degree which lie in the image of the preceding homomorphism.

The next definition is of a notational nature.

Definition (2.1.3). Let 1 denote the graded commutative algebra over $Z_{p}$ which is zero in all positive homogeneous degrees and is isomorphic to $Z_{p}$ in degree zero. If the sequence

$$
1 \rightarrow A \rightarrow B \rightarrow C \rightarrow 1
$$

of commutative graded algebras over $Z_{p}$ is exact, we shall write $B / / A$ for $C$ in accordance with the notation of [8].

(2.2) Keeping the notation and assumption of (2.1), we assume in addition that $\Gamma$ is isomorphic to $Z_{p^{n}}$. Recall that

$$
H^{*}(B)=\left\{\begin{array}{l}
P(y, 1), \quad \text { if } p=2 \text { and } n=1, \\
E(x, 1) \otimes P(y, 2), \quad \text { if } p>2, \text { or } p=2 \text { and } n>1,
\end{array}\right.
$$

where $E(x, 1)$ is the exterior algebra on one generator of degree 1 , while $P(y, 2)$ is the polynomial algebra on one generator of degree 2 . Similarly for $P(y, 1)$. Then,

THEOREM (2.2.1). With the assumptions of the previous paragraph,

(i) $\operatorname{ch}(f) \approx\left\{\begin{array}{l}P(y, 1) /\left(y^{k}\right), k \text { being an integer } \geqq 2, \text { if } p=2 \text { and } n=1 ; \\ E(x, 1) \otimes P(y, 2) /\left(y^{k}\right), k \text { being an integer } \geqq 1, \text { if } p>2 \text {, or } p=2 \\ \text { and } n>1 .\end{array}\right.$ If $\Gamma$ is invariant in $G$, then $\operatorname{ch}(f)$ is a sub-Hopf-algebra of $H^{*}(U)$ and $k$ is a power of $p$.

(ii) $H^{*}(G) / / f^{*} H^{*}(U) \approx E(b, m)$, the exterior algebra with one generator $\bar{b}$ of degree $m, m$ being equal to $k-1$ if $p=2$ and $n=1$, and to $2 k-1$ if $p>2$, or $p=2$ and $n>1$.

(iii) The sequence

$$
1 \rightarrow \operatorname{ch}(f) \rightarrow H^{*}(U) \stackrel{f^{*}}{\rightarrow} H^{*}(G) \rightarrow E(b, m) \rightarrow 1
$$

of graded algebras and homomorphisms is exact.

(2.3) Let $G$ and $U$ be compact connected groups, and suppose that

$$
f: G \rightarrow U
$$


is an epimorphism with a totally disconnected kernel. Thus both $H^{*}(G)$ and $H^{*}(U)$ are connected, commutative, and associative Hopf-algebras. (For the definition of Hopf-algebras and their properties we refer to [8].) Moreover,

$$
f^{*}: H^{*}(U) \rightarrow H^{*}(G),
$$

the homomorphism induced by $f$, is a homomorphism of Hopf-algebras. Therefore, if we denote the space of primitive elements in $H^{*}(U)$ by $V_{u}$ and those in $H^{*}(G)$ by $V_{o}$, we get that

$$
\hat{f} V_{u} \subset V_{o},
$$

where $\hat{f}$ is $f^{*}$ restricted to $V_{u}$. Recall that $V_{u}$ is a graded vector space; i.e., $V_{u}=\sum_{i>0} V_{u}^{i}$, where $V_{u}^{t}$ is the subspace of homogeneous elements of degree $i$. Let us assume that no nonzero elements of even degree exist in $V_{u}$. Then the natural injection $\sigma_{u}: V_{u} \rightarrow H^{*}(U)$ induces a mapping

$$
\sigma_{u}: E\left(V_{u}\right) \rightarrow H^{*}(U)
$$

of Hopf-algebras if the square of every element in $H^{*}(U)$ is zero. The result we are after is

ThEOREM (2.3.1). In addition to the assumptions of the previous paragraph, assume that

$$
\sigma_{u}: E\left(V_{u}\right) \rightarrow H^{*}(U)
$$

is defined and is an isomorphism. Then $V_{0}$ contains no nonzero element of even degree, and the injection $V_{\bullet} \rightarrow H^{*}(G)$ induces

$$
\sigma_{0}: E\left(V_{0}\right) \rightarrow B^{*}(G),
$$

an isomorphism of Hopf-algebras. Further,

(i) $\operatorname{ker} \hat{f} \subset V_{u}^{1}$,

(ii) coker $\hat{f}$ consists of elements of degree 1 ,

(iii) rank $V_{\boldsymbol{o}}=$ rank $V_{u}$.

An interesting feature of this theorem is that $f^{*}$ is determined by its influence on the primitive elements of degree 1.

(2.4) Consider the exact sequence of compact connected groups and homomorphisms,

$$
1 \rightarrow K \stackrel{i}{\rightarrow} G \stackrel{f}{\rightarrow} U \rightarrow 1,
$$

and the induced sequence of cohomology algebras and homomorphisms,

$$
1 \rightarrow B^{*}(U) \stackrel{f^{*}}{\rightarrow} B^{*}(G) \stackrel{i^{*}}{\rightarrow} H^{*}(K) \rightarrow 1 .
$$

It turns out that the problems of describing $i^{*}$ and $f^{*}$ are intimately related. In fact, we shall prove 
TheOREM (2.4.1). Consider the sequence ( $\mathrm{S}^{*}$ ) induced by (S) above. Then $i^{*}$ is an epimorphism if and only if $f^{*}$ is a monomorphism.

One may suspect that $i^{*}$ is always an epimorphism or, equivalently, that $f^{*}$ is always a monomorphism. That this is not necessarily the case is shown by this simple example: Consider the unitary group on two letters $U_{2}$, and let $i: S^{1} \rightarrow U_{2}$ be the imbedding of the circle group $S^{1}$, which takes $e^{i \theta}$ to the diagonal matrix whose nontrivial entries are all equal to $e^{i \theta}$. Then $i\left(S^{1}\right) \cdot S U_{2}$ $=U_{2}$, and $i\left(S^{1}\right) \cap S U_{2} \approx Z_{2}$. Thus we obtain the following exact sequence of compact connected groups and homomorphisms:

$$
1 \rightarrow S^{1} \stackrel{i}{\rightarrow} U_{2} \rightarrow U_{2} / i\left(S^{1}\right)=S U_{2} / Z_{2} \rightarrow 1 .
$$

The induced sequence of the cohomology algebras mod 2 fails to be exact because $H^{*}\left(U_{2} ; Z_{2}\right)$ is an exterior algebra, whereas $H^{*}\left(S U_{2} / Z_{2} ; Z_{2}\right)$ is a truncated polynomial algebra.

The analysis of $\left(\mathrm{S}^{*}\right)$ will be taken up in some detail in $\$ \S 8$ and 9 . We shall see that it is possible to construct a split exact sequence $\left(\mathrm{S}^{\prime}\right)$ covered by $(\mathrm{S})$. The problem is thereby reduced to examining what happens to the exactness of the induced cohomology sequence $\left(\mathrm{S}^{\prime *}\right)$ on "going up." It will be seen that whatever makes the example given above fail to be exact is, roughly speaking, all that can go wrong. In particular, we shall prove

Theorem (2.4.2). Consider the sequence $\left(\mathrm{S}^{*}\right)$ given above. Suppose that the space of primitive elements $V_{u}$ of $H^{*}(U)$ has no nonzero elements of even degree and that the natural map,

$$
\sigma_{u}: E\left(V_{u}\right) \rightarrow H^{*}(U)
$$

is an isomorphism. Then

(i) $i^{*}$ is an epimorphism or, equivalently, $f^{*}$ is a monomorphism, and

(ii) $H^{*}(G) / / H^{*}(U)=H^{*}(K)$, as Hopf-algebras.

We shall deduce from these theorems a few interesting relationships between the cohomology algebra of a compact connected group and its topology. A precise statement of these results and their proofs will be given in $\S 3$. The proofs of the main theorems will be given in $\$ \S 6,7,8$, and 9 .

REMARKS (2.5). Borel, in $[1, \S 10]$, proves special cases of Theorem (2.2.1). Our proof is quite different from his and our result is more general. Also, W. Browder announced in [3] a weaker result for $H$-spaces whose cohomology is suitably restricted; his method is different from ours.

3. Corollaries. In this section we give some of the applications of our main theorems. We shall first state and discuss them, deferring the more complicated proofs to the later subsections. The discussion covers three main topics: the characterization of the commutativity of the group in terms of its cohomology algebra, finite-dimensional groups and the resemblance of their 
cohomology to that of Lie groups, and the class of those groups whose cohomology is an exterior algebra (it will be seen that this class is closed under many useful operations); these topics are taken up in (3.1), (3.2) and (3.3), respectively.

Note that, although we have restricted ourselves so far to compact connected groups, many of the results of this section are true also for locally compact connected groups, since a group of this kind is of the same homotopy type as any of its maximal compact subgroups $[9, \S 4.13]$.

(3.1) Consider the compact connected group $G$, and let $H^{*}\left(G ; Z_{p}\right)$ be its cohomology algebra with coefficients in $Z_{p}$, the prime field of characteristic $p$.

An old result of E. Cartan's states that $G$ is abelian if and only if $H^{*}\left(G ; Z_{0}\right)$, the rational cohomology algebra of $G$, has no primitive elements of degree $3[5]$. When $p>0$ the situation is more complicated; for instance, if $p=2$, the result is no longer true; for the group $\mathrm{SO}_{3}$ is not commutative although its cohomology mod 2 has no primitive elements of degree 3 . Thus a good criterion for the commutativity of $G$ must exclude more than the primitive elements of degree 3 . So let $V_{p}$ denote $H^{1}\left(G ; Z_{p}\right)$. Our first result is

TheOREM (3.1.1). The natural injection of $V_{p}$ in $H^{*}\left(G ; Z_{p}\right)$ extends to an isomorphism of Hopf-algebras, $\sigma: E\left(V_{p}\right) \rightarrow H^{*}\left(G ; Z_{p}\right)$, if and only if $G$ is abelian.

It is also possible to obtain a criterion for $G$ to be abelian in terms of $H^{1}(G ; Z)$. Let $V$ stand for $H^{1}(G ; Z)$; then the natural injection of $V$ into $H^{*}(G ; Z)$ extends to a homomorphism of algebras

$$
\sigma: E(V) \rightarrow H^{*}(G ; Z),
$$

since the square of every element of $H^{*}(G ; Z)$ of degree 1 is always zero. The criterion is

COROLlaRy (3.1.2). With the notation being as in the previous paragraph, $G$ is abelian if and only if $\sigma$ is an isomorphism.

Proof. Suppose that $G$ is abelian. Then there is an inverse system of tori $\left\{T_{\alpha}, \phi_{\alpha \beta}\right\}$ such that

$$
G=\underbrace{\lim }_{\alpha} T_{\alpha} .
$$

Denote $H^{1}\left(T_{\alpha}, Z\right)$ by $V_{\alpha}$. Then the homomorphism $\sigma_{\alpha}: E\left(V_{\alpha}\right) \rightarrow H^{*}\left(T_{\alpha} ; Z\right)$ is an isomorphism for every $\alpha$. We also know that

$$
V=\frac{\lim }{\alpha} V_{\alpha}
$$

and, hence,

$$
E(V)=\frac{\lim }{\longleftarrow} E\left(V_{\alpha}\right) .
$$


Therefore, $\sigma: E(V) \rightarrow H^{*}(G ; Z)$ is an isomorphism.

Conversely, suppose that $\sigma: E(V) \rightarrow H^{*}(G ; Z)$ is an isomorphism. Since $V=H^{1}(G ; Z)$ is free of torsion, it follows that $E(V)$ is too. Hence $H^{*}(G ; Z)$ is free of torsion. Therefore, $H^{*}\left(G ; Z_{p}\right)=H^{*}(G ; Z) \otimes Z_{p} ; E\left(V \otimes Z_{p}\right)$ $=E(V) \otimes Z_{p} ;$ and $H^{1}\left(G ; Z_{p}\right)=V \otimes Z_{p}$. This means that $\sigma_{p}: E\left(V_{p}\right) \rightarrow H^{*}\left(G ; Z_{p}\right)$ is an isomorphism. By Theorem (3.1.1), $G$ is abelian. Q.E.D.

(3.2) It is well known that for an arbitrary compact connected space $X$, neither the vanishing of its cohomology groups in arbitrarily high dimensions, nor the condition that it be finitely generated in every homogeneous dimension, constitutes sufficient reason for the finite-dimensionality of the space. If, in addition, $X$ is a group, it turns out that either of these conditions implies the finite-dimensionality of $X$, except in very special cases. The following theorem describes the situation completely.

THEOREM (3.2.1). Let $G$ be a compact connected group and p, a prime. If $G$ is finite-dimensional, then there is an integer $N$ such that

(i) $H^{i}\left(G ; Z_{p}\right)=0$, for all $i>N$, and

(ii) $H^{*}\left(G ; Z_{p}\right)$ is finitely generated in every homogeneous degree.

Conversely, if either (i) or (ii) is satisfied and no element of $\operatorname{char}\left(G^{c}\right)$, the character group of the connected component of the identity of the center of $G$, is infinitely divisible by $p$, then $G$ is finite-dimensional. (In particular, if $p=0$, then (i) or (ii) will imply the finite-dimensionality of $G$.)

The restriction on $\operatorname{char}\left(G^{c}\right)$ is necessary as shown by the following example: let $S^{1}$ be the group of complex numbers $z$ of absolute value 1 , and consider the inverse system,

$$
\ldots \stackrel{f}{\rightarrow} S^{1} \stackrel{f}{\rightarrow} S^{1} \stackrel{f}{\rightarrow} \ldots \stackrel{f}{\rightarrow} S^{1}
$$

where $f$ is the homomorphism taking $z$ into $z^{p}$ for some fixed prime $p$; the inverse limit $\sum_{p}$ is the so-called $p$-adic solenoid, and $H^{k}\left(\sum_{p} ; Z_{p}\right)=0$ for all $k>0$. Put $G$ equal to the cartesian product of $\sum_{p}$ with itself an infinite number of times; then $H^{k}\left(G ; Z_{p}\right)$ is zero for all $k>0$, but $G$ is not finitedimensional.

An easy corollary of Theorem (3.2.1) is the following.

CoRollary (3.2.2). Let $G$ be a compact connected group. Then, if there exist an integer $N$ such that $H^{i}(G ; Z)=0$, for all $i>N$, it follows that $G$ is separable metric.

There are certain distinctive properties that the integral cohomology algebras of compact connected Lie groups have:e.g., the existence of a fundamental class and the freeness of the first integral cohomology groups. We shall show that, among compact groups, only Lie groups exhibit these properties. More precisely, 
Proposition (3.2.3). Let $G$ be a compact connected group. Then $G$ is a Lie group if and only if there exists an integer $N$ such that $H^{i}(G ; Z)=0$, for all $i>N$, and $H^{N}(G ; Z)=Z$. Further, $N=\operatorname{dim} G$.

Proposition (3.2.4). Let $G$ be a compact connected group. Then $G$ is a Lie group if and only if there is an integer $N$ such that $H^{i}(G ; Z)=0$, for $i>N$, and $H^{1}(G ; Z)$ is free.

Proofs for these propositions are given in (3.6).

(3.3) Let us consider now the following class of groups.

Definition (3.3.1). If $p$ is a prime, we denote by $\mathfrak{C}_{p}$ the class of those compact connected groups $G$ such that $H^{*}\left(G ; Z_{p}\right)$ is generated by elements of odd degree and height 2.

The Samelson-Leray Theorem [8, Theorem (4.10)] allows us to give an alternative definition.

Definition (3.3.2). Let $p$ be a prime and $G$, a compact connected group; denote by $V_{0}$ the spaces of primitive elements of $H^{*}\left(G ; Z_{p}\right)$. By $\mathfrak{C}_{p}$ we denote the class of compact connected groups $G$ such that $V_{0}$ has no nontrivial elements of even degree and the natural injection of $V_{g}$ in $H^{*}\left(G ; Z_{p}\right)$ extends to an isomorphism, $\sigma_{\vartheta}: E\left(V_{\imath}\right) \rightarrow H^{*}\left(G ; Z_{p}\right)$, of Hopf-algebras.

If $G$ is a compact connected Lie group, then a result of Borel's says that it is in $\mathfrak{e}_{p}$ if and only if $H^{*}(G ; Z)$ is free of $p$-torsion. The following result summarizes the main properties of $\mathfrak{e}_{p}$.

Theorem (3.3.3). The class $\mathfrak{C}_{p}$, defined above, has the following properties:

(i) it contains all abelian groups;

(ii) if $G \in \mathfrak{e}_{p}$, each closed connected invariant subgroup of $G$ is in $\mathfrak{e}_{p}$;

(iii) if $1 \rightarrow K \rightarrow G \rightarrow U \rightarrow 1$ is exact and $K$ and $U$ are in $\mathfrak{e}_{p}$, then $G$ is in $\mathfrak{e}_{p}$;

(iv) if $1 \rightarrow \Gamma \rightarrow G \rightarrow U \rightarrow 1$ is exact, $\Gamma$ is totally disconnected, $G$ is compact connected, and $U$ is in $\mathfrak{e}_{p}$, then $G$ is in $\mathfrak{C}_{p}$.

(3.4) Before proceeding to the proofs of the results of the previous theorems, we recall the results of E. Cartan and Van Kampen on the structure of compact connected groups [11].

Denote by $G^{c}$ the connected component of the identity of the center of $G$; and by $G^{s}$, the closure of the commutator subgroup of $G$. Both $G^{c}$ and $G^{*}$ are closed normal and connected subgroups of $G$; when $G$ is finite-dimensional, $G^{\boldsymbol{s}}$ is just the commutator subgroup of $G$. In general $G^{\boldsymbol{s}}$ is larger. $G^{\boldsymbol{s}}$ can be represented in a unique way, up to an automorphism, in the form $\hat{G}^{s} / D$, where $\hat{G}^{*}$ is a product, in general infinite, of simple, simply-connected compact Lie groups and $D$ is a totally-disconnected subgroup of the center. Let us remark that $D$ is finite when the product is finite. Denote by $\hat{G}$ the group $\hat{G}^{*} \times G^{c}$. Let $\psi_{0}: \hat{G}^{s} \rightarrow \hat{G}^{o} / D=G^{s}$ be the natural epimorphism. Define $\phi_{0}: \hat{G} \rightarrow G$ by putting $\phi_{0}(x, y)=\psi_{0}(x) \cdot y$, where $x, y$ are arbitrary elements of $\hat{G}^{s}$ and $G^{c}$, respectively. 
Then $\phi_{0}$ is an epimorphism which, when restricted to the first factor, reduces to $\psi_{0}$, while it reduces to the identity when restricted to the second factor.

(3.5) We shall now prove Theorem (3.1.1). The proof that if $G$ is abelian then $\sigma: E\left(V_{p}\right) \rightarrow H^{*}\left(G ; Z_{p}\right)$ is an isomorphism runs along the lines of the argument in (3.1.2); we therefore shall not reproduce it. So assume that $\sigma: E\left(V_{p}\right)$ $\rightarrow H^{*}\left(G ; Z_{p}\right)$ is defined and is an isomorphism of Hopf-algebras. Consider the group $\hat{G}$, described in (3.4), and its epimorphism, $\phi_{0}: \hat{G} \rightarrow G$. Since ker $\phi_{0}$ is totally disconnected, Theorem (2.3.1) implies that $\hat{V}_{p}$, the space of primitive elements of $H^{*}\left(\hat{G} ; Z_{p}\right)$, consists of one-dimensional elements only. Since, by definition, $\hat{G}=\left(\prod_{i} G_{i}\right) \times G^{c}$, where each $G_{i}$ is a simple, simply-connected and compact Lie group, to show that $G$ is abelian we need only show that if the first factor of $\hat{G}$ were not trivial, then $\hat{V}_{p}$ would have nontrivial elements of degree 3. But this follows from the following proposition, which is an easy consequence of a result of Bott and Samelson's [2].

Proposition (3.5.1). Suppose $G$ is a simple, simply-connected and compact Lie group. Then $H^{3}(G ; Z) \neq 0$ and consists entirely of primitive elements.

(3.6) For the proofs of the results of (3.2), we need the following lemmas. Let $G$ and $U$ be compact connected groups, and let $f: G \rightarrow U$ be an epimorphism whose kernel is isomorphic to $Z_{p}$. Since $f$ is a homomorphism, $\operatorname{ch}(f)$ is a sub-Hopf-algebra of $H^{*}(U)$ and $\operatorname{im} f^{*}$ is a sub-Hopf-algebra of $H^{*}(G)$. Therefore, by part (iii) of Theorem (2.2.1), we get the following two exact sequences of Hopf-algebras and homomorphisms:

$$
\begin{array}{ll}
\left(\mathrm{S}_{1}\right): & 1 \rightarrow \operatorname{ch}(f) \rightarrow H^{*}(U) \rightarrow \operatorname{im} f^{*} \rightarrow 1, \\
\left(\mathrm{~S}_{2}\right): & 1 \rightarrow \operatorname{im} f^{*} \rightarrow H^{*}(G) \rightarrow E(b, m) \rightarrow 1 .
\end{array}
$$

Hence, by $[8$, Theorem (2.5)],

$$
\begin{aligned}
& H^{*}(U)=\operatorname{cf}(f) \otimes \operatorname{im} f^{*} \text { as modules over } \operatorname{ch}(f), \text { and } \\
& H^{*}(G)=\operatorname{im} f^{*} \otimes E(b, m) \text { as modules over } \operatorname{im} f^{*} .
\end{aligned}
$$

Notice that $\operatorname{ch}(f)$ has no nontrivial elements of degree greater than $m$. Hence,

LemMa (3.6.1). If $H^{i}\left(U ; Z_{p}\right)=0$, for all $i>$ some integer $N$, then $H^{i}\left(G ; Z_{p}\right)$ $=0$, for all $i>N$.

Suppose now that $H^{*}\left(G ; Z_{p}\right)$ and $H^{*}\left(U ; Z_{p}\right)$ are finitely generated in every homogeneous degree. Write $P_{p}(G ; t), P_{p}(U ; t), P_{p}(f ; t)$, and $P_{p}(\chi ; t)$ for the Poincaré series of $H^{*}\left(G ; Z_{p}\right), H^{*}\left(U ; Z_{p}\right)$, im $f^{*}$, and $\operatorname{ch}(f)$, respectively; i.e., $P_{p}(G ; t)$ is the formal sum $\sum_{i \geq 0} a_{i} t^{i}$, where $a_{i}=\operatorname{rank} H^{i}\left(G ; Z_{p}\right)$. Similarly for $P_{p}(U ; t), \operatorname{im} f^{*}$, and $\operatorname{ch}(f)$. By the decomposition $\left(^{*}\right)$ given above we get

$$
P_{p}(U ; t)=P_{p}(\chi ; t) \cdot P_{p}(f ; t),
$$

and 


$$
P_{p}(G ; t)=P_{p}(f ; t) \cdot\left(1+t^{m}\right)
$$

But

$$
P_{p}(\chi ; t)= \begin{cases}\left(1+t+t^{2}+\cdots+t^{k-1}\right), & \text { if } p=2 \\ (1+t)\left(t+t^{2}+\cdots+t^{2 k-2}\right), & \text { if } p>2\end{cases}
$$

and $m=k-1$ when $p=2$ and $2 k-1$ when $p>2$. Hence

Lemma (3.6.2). $P_{p}(G ; t) \leqq P_{p}(U ; t)$.

Now we can proceed to give the proofs for (3.2).

Proof of Theorem (3.2.1). Suppose that $G$ is a finite-dimensional compact connected group. Then there is an epimorphism $f: G \rightarrow U$ where $U$ is a compact connected Lie group and ker $f$ is totally disconnected. Factor $f$ into an inverse limit of epimorphisms with finite kernels and apply (3.6.1). This proves the necessity of the assertion.

Next assume that $H^{*}\left(G ; Z_{p}\right)$ is finitely generated in every homogeneous dimension. Observe that the following commutative diagram has exact rows:

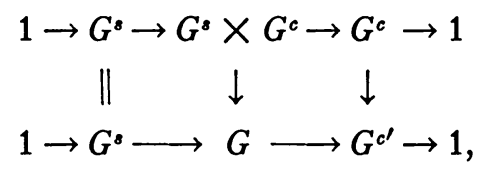

where $G^{s}$ and $G^{c}$ have the same meaning given them in (3.4) and $G^{\prime \prime}$ is a connected abelian group. By Theorem (2.4.2), we know that $H^{*}\left(G^{s} ; Z_{p}\right)$ is a quotient of $H^{*}\left(G ; Z_{p}\right)$ and, hence, is finitely generated in every homogeneous dimension. Applying (3.6.2) to the epimorphism $\psi_{0}: \hat{G}^{s} \rightarrow G^{s}$ (see (3.4)), we get that $H^{*}\left(\hat{G}^{s} ; Z_{p}\right)$ is also finitely generated in every homogeneous dimension. Hence, by (3.5.1), $\hat{G}^{\boldsymbol{s}}$ is finite-dimensional. Similarly, $H^{*}\left(G^{c} ; Z_{p}\right)$ is finitely generated in èvery homogeneous dimension. If no element of $H^{1}\left(G^{c} ; Z\right)$ (which is, by a classical result, equal to $\operatorname{char}\left(G^{c}\right)$ ) is infinitely divisible by $p$, it follows that $\operatorname{char}\left(G^{c}\right)$ is of finite rank and, hence, that $G^{c}$ is finite-dimensional. Since both $\hat{G}^{s}$ and $G^{c}$ are finite-dimensional, it follows that $G$ itself is finite-dimensional.

The proof of the fact that the vanishing of the cohomology groups mod $p$ of $G$ in arbitrarily high dimensions, together with the condition on $\operatorname{char}\left(G^{c}\right)$, imply the finite-dimensionality of $G$ is similar. Q.E.D.

Proof of Corollary (3.2.2). Let $G$ be a compact connected group, and suppose that there exists an integer $N$ such that $H^{i}(G ; Z)=0$ for $i>N$. Then the universal coefficient theorem implies that $H^{i}\left(G ; Z_{0}\right)=0$ for $i>N$, where $Z_{0}$ is the field of rational numbers. Thus, by Theorem (3.2.1), $G$ is finite-dimensional. First notice that, since $G$ is compact, its metrisability follows as soon as we establish its separability. So consider now the group $\hat{G}$, associated with $G$, and the epimorphism $\phi_{g}: \hat{G} \rightarrow G$ (see (3.4)). Since $\phi_{0}$ is an open map, to prove that $G$ is separable it is enough to prove that $\hat{G}$ itself is separable. By defini- 
tion, $\hat{G}=\hat{G}^{s} \times G^{c}$, where $\hat{G}^{s}$ is a compact connected Lie group and $G^{c}$ is the connected component of the center of $G$. Thus it is enough to show that $G^{c}$ is separable or, equivalently, that $\operatorname{char}\left(G^{c}\right)$ is countable. First, we know that $\operatorname{char}\left(G^{c}\right)$ is of finite rank, since $G^{c}$ is finite-dimensional. Hence $\operatorname{char}\left(G^{c}\right) \otimes_{z} Z_{0}$ is a finite-dimensional vector space over $Z_{0}$ and is, therefore, countable. Further, since $G^{c}$ is connected, we know that $\operatorname{char}\left(G^{c}\right)$ is free of torsion. Thus the natural monomorphism $Z \rightarrow Z_{0}$ induces a monomorphism $\operatorname{char}\left(G^{c}\right)$ $\rightarrow \operatorname{char}\left(G^{c}\right) \otimes_{z} Z_{0}$. Therefore $\operatorname{char}\left(G^{c}\right)$ is countable. Q.E.D.

Proof of Proposition (3.2.3). Let $G$ be a compact connected group, and suppose that there exists an integer $N$ such that $H^{i}(G ; Z)=0$, for all $i>N$, and $H^{N}(G ; Z)=Z$. First of all, Theorem (3.2.1) tells us that $G$ is finite-dimensional. Next, by the Peter-Weyl Theorem, $G$ is the inverse limit of compact connected Lie groups. Hence, there exists a compact connected Lie group $G^{\prime}$ and an epimorphism $f: G \rightarrow G^{\prime}$ with a totally disconnected kernel such that $f^{*}: H^{N}\left(G^{\prime} ; Z\right) \rightarrow H^{N}(G ; Z)$ is an isomorphism. We can also find an inverse system of compact connected Lie groups $G_{\alpha}$, and, for each $\alpha$, an epimorphism $f_{\alpha}: G_{\alpha} \rightarrow G^{\prime}$ with a finite kernel such that

$$
G=\frac{\lim }{\alpha} G_{\alpha} \text { and } \underset{\alpha}{\stackrel{\lim }{\longleftarrow}} f_{\alpha}=f .
$$

Therefore $f_{\alpha}^{*}: H^{N}\left(G^{\prime} ; Z\right) \rightarrow H^{N}\left(G_{\alpha} ; Z\right)$ is an isomorphism for all $\alpha$, since $f$ factors through each $G_{\alpha}$. Hence, for all $\alpha$ and every prime $p, f_{\alpha}^{*}: H^{N}\left(G^{\prime} ; Z_{p}\right)$ $\rightarrow H^{N}\left(G_{\alpha} ; Z_{p}\right)$ is an isomorphism. This implies that ker $f_{\alpha}=1$, for, otherwise, if $p$ divided the order of $\operatorname{ker} f_{\alpha}$, Theorem (2.2.1) would imply that the fundamental class of $G_{\alpha}$ does not lie in $\operatorname{im} f_{\alpha}^{*}$. Q.E.D.

Proof of Proposition (3.2.4). Let $G$ be a compact connected group, and assume that there exists an integer $N$ such that $H^{i}(G ; Z)=\{0\}$, for $i>N$. Suppose, moreover, that $H^{1}(G ; Z)$ is free. Observe, first of all, that, by virtue of Theorem (3.2.1), $G$ is finite-dimensional. Hence $H^{1}(G ; Z)$ is finitely generated. Consider now the canonical group $\hat{G}$ associated with $G$, and let $\phi_{g}: \hat{G} \rightarrow G$ be its natural epimorphism (see (3.4)). Then $\operatorname{ker} \phi_{0}$ is finite, of order $M$, say. We claim

Lemma. $A=H^{1}(\hat{G} ; Z) / \phi_{0}^{*} H^{1}(G ; Z)$ is a finite group.

Proof. Using the transfer homomorphism $\tau: H^{1}(\hat{G} ; Z) \rightarrow H^{1}(G ; Z)[4]$, we see that $\phi_{0}^{*}: H^{1}(G ; Z) \rightarrow H^{1}(\hat{G} ; Z)$ is a monomorphism and that the order of every element of $H^{1}(\hat{G} ; Z) / \phi_{g}^{*} H^{1}(G ; Z)$ divides $M$. Suppose now that $p^{m}$ is the highest power of $p$ dividing $M$, where $p$ is a prime. By tensoring the exact sequence

$$
0 \rightarrow H^{1}(G ; Z) \stackrel{\phi_{g}^{*}}{\longrightarrow} H^{1}(\hat{G} ; Z) \rightarrow A \rightarrow 0
$$

by $Z_{p}{ }^{m}$, we get the exact sequence 
$0 \rightarrow \operatorname{Tor}\left(A ; Z_{p^{m}}\right) \rightarrow H^{1}(G ; Z) \otimes Z_{p^{m}} \stackrel{\phi_{0}^{*} \otimes 1}{\longrightarrow} H^{1}(\hat{G} ; Z) \otimes Z_{p^{m}} \rightarrow A \otimes Z_{p^{m}} \rightarrow 0$.

Since $H^{1}(G ; Z)$ is finitely generated and free, $H^{1}(G ; Z) \otimes Z_{p^{m}}$ is isomorphic to a finite direct sum of copies of $Z_{p^{m}}$ and, hence, is finite. Hence $\operatorname{Tor}\left(A ; Z_{p^{m}}\right)$ (which is the subgroup of $A$ consisting of those elements whose orders divide $\left.p^{m}\right)$ is also finite. But $p$ is an arbitrary prime dividing $M$. Hence $A$ is finite. Q.E.D.

Thus $H^{1}(\hat{G} ; Z)$ is a finitely generated free group. But $H^{1}(\hat{G} ; Z)=H^{1}\left(G^{c} ; Z\right)$, where $G^{c}$ is the connected component of the center of $G$. Hence, $H^{1}\left(G^{c} ; Z\right)$ and, thus, $\operatorname{char}\left(G^{c}\right)$, is finitely generated and free. Hence $G^{c}$ is a torus. Therefore, $\hat{G}$ is a Lie group, and, hence, $G$ is a Lie group [11]. Q.E.D.

(3.7) We shall give here the proof of Theorem (3.3.3). Parts (i) and (iv) of the theorem are portions of the statements of Theorems (3.1.1) and (2.3.1), respectively; thus we need prove only (ii) and (iii). The latter is a consequence of an essentially algebraic result regarding extensions of Hopf-algebras. If $A$ is a Hopf-algebra, we shall denote by $p(A)$ and $q(A)$ the spaces of primitive elements and indecomposable elements of $A$, respectively [8, §3].

Proposition (3.7.1). Consider the exact sequence

$$
(\mathrm{S}): 1 \rightarrow A \stackrel{i}{\rightarrow} B \stackrel{j}{\rightarrow} C \rightarrow 1
$$

of commutative, associative and connected Hopf-algebras over the field $Z_{p}$. If $p(A)=q(A)$ and $p(C)=q(C)$ and each has no nonzero elements of even degree, then $p(B)$ is isomorphic to $q(B)$ and has no nonzero elements of even degree. Moreover, the sequence

$$
0 \rightarrow p(A) \rightarrow p(B) \rightarrow p(C) \rightarrow 0
$$

is exact.

Proof. The sequence $(\mathrm{S})$ induces the following commutative ladder of vector spaces with exact rows $[8$, Theorem (3.6)]:

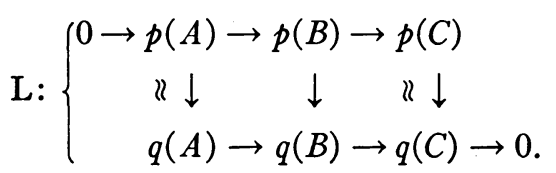

To prove the proposition it is enough, by $[8$, Theorem (4.10)], to show that $B$ is generated by elements of odd degree and height 2 . So choose a homomorphism $\alpha: C \rightarrow B$ such that $j \circ \alpha=1$. Denote by $L$ the subspace $i p(A)+\alpha p(C)$. It is easy to see that the natural map $B \rightarrow B / \bar{B}^{2}=q(B)$ maps $L$ on $q(B)$; i.e., $L$ generates $B$. Moreover, $L$ contains no nontrivial elements of even degree. We want to show that every element of $L$ has height 2 . Since the case $p>2$ gives no trouble, assume $p=2$. In fact, it is just as easy to show that every element of $B$ has height 2 . So suppose $x \in B_{1}$. Then $x$ is primitive and, 
hence, $x^{2}$ is also primitive. Thus $x^{2}=0$, because $p(B)$ has no nontrivial elements of even degree. Assume now that it has been shown that if $x \in B_{m}$ and $m<n$, then $x^{2}=0$. Take $x \in B_{n}$. Then, by the induction hypothesis, $x^{2}$ is primitive. But deg $x^{2}$ is even. Hence $x^{2}=0$. Q.E.D.

It is clear that Proposition (3.7.1) implies part (iii) of Theorem (3.3.3). So let us now turn to the proof of part (ii). As a first step, we assert

Proposition (3.7.2). Let $G$ be a compact connected group. Then $G$ is in $\mathfrak{C}_{p}$ if and only if $G^{*}$ is in $\mathcal{C}_{p}$ (see (3.4)).

Proof. Consider the commutative diagram

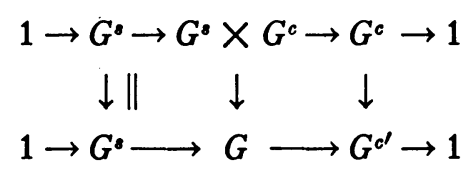

and apply Theorem (2.4.2).

Now suppose $G$ is a compact connected group in $\mathfrak{C}_{p}$, and let $K$ be an invariant subgroup of $G$. By (3.7.2), $K \in \mathfrak{e}_{p}$ if and only if $K^{\bullet} \in \mathfrak{e}_{p}$. So consider the following commutative diagram of compact connected groups and homomorphisms (see (3.4)):

$$
\mathrm{D}:\left\{\begin{aligned}
1 \rightarrow \hat{K}^{s} & \stackrel{\hat{\imath}}{\rightarrow} \hat{G}^{s} \rightarrow \hat{G}^{s} / \hat{K}^{s} \rightarrow 1 \\
\downarrow & \downarrow \downarrow \psi_{o} \quad \downarrow \\
1 \rightarrow K^{s} \rightarrow & G^{s} \rightarrow G^{s} / K^{s} \rightarrow 1 .
\end{aligned}\right.
$$

Dividing the upper row by the subgroup $\Gamma_{k}=\operatorname{ker} \psi_{g} \cap \hat{K}^{*}$, we get

$$
\begin{gathered}
1-\hat{K}^{s}-\hat{G}^{s}-\hat{G}^{s} / \hat{K}^{s}-1 \\
\downarrow \\
\downarrow \\
1-K^{s}-\hat{G}^{s} / \Gamma_{k}-\hat{G}^{s} / \hat{K}^{s}-1 \\
\| \quad{ }^{\downarrow} \quad \\
1-K^{s}-G^{s}-G^{s} / K^{s}-1 .
\end{gathered}
$$

The middle row splits, and $\hat{G}^{\imath} / \Gamma_{k} \in \mathcal{C}_{p}$, by Theorem (2.3.1) and Proposition (3.7.2). Hence $K^{\bullet} \in \mathfrak{C}_{p}$. Q.E.D.

4. The category of principal fibre spaces. The main purpose of this and the succeeding sections is to set the stage for the proofs of $\$ 2$. The category of principal fibre spaces is described briefly, and a few simple propositions about this category will be formulated. They will be used in $\$ 5$.

(4.1) We shall limit ourselves to Hausdorff spaces and compact groups throughout. So let $E$ be a Hausdorff space and $G$, a compact group. Recall that $G$ is said to act on $E$ if and only if there exists a continuous map, $\phi: E \times G \rightarrow E$, such that for all $x \in E, \phi(x, 1)=x$ where $1 \in G$ is the identity and 
$\phi\left(x, g g^{\prime}\right)=\phi\left(\phi(x, g), g^{\prime}\right)$ for all $g, g^{\prime} \in G$. If, in addition, $\phi(x, g) \neq x$ whenever $g \neq 1 \in G$, the action is said to be free. In this case $E$ is called a principal fibre space for $G$. Denote the decomposition space $E / G$ by $B$, and the natural map $E \rightarrow B$ by $f . B$ is a Hausdorff space, and it is called the base, while $f$ is called the fibre map. Of course $f$ need not satisfy the covering homotopy theorem. This is all right because we shall be using the Leray spectral sequence.

The objects of the category $P \mathcal{F}$ are the triples $(E, \phi, G)$, where $E$ is a principal fibre space for $G$. A map of $(E, \phi, G)$ into $\left(E^{\prime}, \phi^{\prime}, G^{\prime}\right)$ is a pair of maps $(\mu, \lambda)$, where $\mu: E \rightarrow E^{\prime}$ and $\lambda: G \rightarrow G^{\prime}$ is a homomorphism such that $\mu \circ \phi$ $=\phi^{\prime} \circ(\mu \times \lambda)$. Instead of $(E, \phi, G)$ it is sometimes convenient to write $f: E \rightarrow B$, where $B$ is the base space and $f$, the fibre map.

ExAmples (4.1.1). (i) The principal fibre bundles belong to PF.

(ii) The total space is a topological group $G$; the group is a compact subgroup $K$; and the action is by translation on the right. Write $(G, \phi, K)$ for this fibre space. $B$ is $G / K$. Such fibrations need not be locally trivial. For example, suppose $G^{\prime}$ is a compact connected Lie group and $K^{\prime}$, a closed subgroup of $G^{\prime}$ such that the fibre bundle $G^{\prime} \rightarrow G^{\prime} / K^{\prime}$ is not trivial. Denote by $G$ the cartesian product of $G^{\prime}$ with itself infinitely many times; and by $K$, the cartesian product of $K^{\prime}$ with itself as many times. Then the fibration for which the total space is $G$ and for which the group is $K$ acting on $G$ coordinatewise is not locally trivial.

(iii) Let $G$ be a compact group, and assume that $\Gamma$ is a finite abelian subgroup of $G$. Denote by $E$ the infinite join of $\Gamma$ with itself. With the weak topology, $E$ is a regular CW-complex which serves as a locally trivial fibre bundle for $\Gamma$. Put $E / \Gamma=B$. Consider now the space $E \times_{\Gamma} G$, i.e., the decomposition space $E \times G / \Gamma$ where $\Gamma$ is made to act on $E \times G$ via the diagonal map. Then the multiplication on $G, \phi: G \times G \rightarrow G$, induces a mapping $1 \times \phi: E \times G \times G$ $\rightarrow E \times G$ which passes to a mapping $1 \times_{\Gamma} \phi:\left(E \times_{\Gamma} G\right) \times G \rightarrow E \times_{\Gamma} G$. In this fashion $E \times_{\Gamma} G$ becomes a principal fibre space for $G$. Moreover, $E \times_{\Gamma} G / G$ $=B$, and the fibre map $E \times_{\Gamma} G \rightarrow B$ is just the map $\bar{\beta}: E \times_{\Gamma} G \rightarrow B$ induced by the composition of the projection $E \times G \rightarrow E$ and the locally trivial fibring $E \rightarrow B$. We shall write $\left(E \times_{\Gamma} G, 1 \times_{\Gamma} \phi, G\right)$ for this fibre space.

Let $\left\{\left(E_{\alpha}, \phi_{\alpha}, G_{\alpha}\right) \mid \alpha \in J\right\}$ be a set of principal fibre spaces. Then $E=\prod_{\alpha} E_{\alpha}$ is a principal fibre space for $G=\prod_{\alpha} G_{\alpha}$, the action being defined by $\phi=\prod_{\alpha} \phi_{\alpha}$. Thus

(4.1.2) The category PF is closed under infinite products.

It is just as easy to prove that:

(4.1.3) The category $P \mathcal{F}$ is closed under inverse limits, and the functor which assigns $B=E / G$ to $(E, \phi, G)$ is continuous when restricted to the subcategory of fibrations for which the total spaces are compact.

(4.2) The examples of (4.1.1) are the fibrations which occur in our work. Those which are locally trivial and fibred by compact Lie groups are the best behaved. We shall see that the other examples are just as nice. 
Proposition (4.2.1). Consider the fibration $(G, \phi, K)$ where $G$ is a compact group; $K$, a closed subgroup of $G$; and $K$ acts on $G$ by translations on the right (see (ii) of (4.1.1)). Then $(G, \phi, K)$ is the inverse limit of fibrations of compact Lie groups by closed subgroups.

The proof is a fairly simple application of the Peter-Weyl Theorem.

5. Spectral sequences and Leray's operators. Let $f: E \rightarrow B$ be a principal fibre space for the compact connected group $G$, and let $Z_{p}$ be the prime field of characteristic $p$. It will be tacitly assumed, unless otherwise mentioned, that the cohomology groups have their coefficients in $Z_{p}$. Consider the cohomology spectral sequence $\left\{E_{r}(f), r \geqq 2\right\}$ of the map $f$. For its definition and functorial properties see $[6, \S 4.17]$. Recall that

$$
E_{2}(f)=B^{*}\left(B ; \mathcal{C}^{*}(G)\right),
$$

where the cohomology theory used is the sheaf-theoretic one with closed supports, and the sheaf of coefficients $\mathfrak{K C}^{*}(G)$ is the sheaf generated by the presheaf which assigns to an open set $V \subset B$ the group $H^{*}\left(f^{-1} V\right)$. This spectral sequence is, however, of little use unless we know that (i) the Cech cohomology groups of $E$ and $B$ agree with their sheaf-theoretic cohomology with closed supports and (ii) the sheaf $\mathfrak{K}^{*}(G)$ has the cohomology groups of the fibres for stalks and is simple and $E_{2}(f)=H^{*}(B) \otimes H^{*}(G)$. The propositions of (5.1) and (5.2) below will prove (i) and (ii) for the fibre spaces needed for the proof of our main results.

The latter part of this section is devoted to the description of the operators of Leray. Our exposition, like that of [1], depends on the solution of a problem of the Künneth type: namely, the relating of $E_{\mathbf{r}}(f)$ and $E_{\boldsymbol{r}}\left(f^{\prime}\right)$ with $E_{r}\left(f \times f^{\prime}\right)$, where $f \times f^{\prime}: E \times E^{\prime} \rightarrow B \times B^{\prime}$ is the product of the fibrations $f: E \rightarrow B$ and $f^{\prime}: E^{\prime} \rightarrow B^{\prime}$. This is done in (5.3).

(5.1) Let $G$ be a compact connected group and $\Gamma$, a finite abelian subgroup of $G$; and write $U$ for the coset space $G / \Gamma$ and $f$ for the natural map $G \rightarrow U$. Suppose also that $\gamma: E \rightarrow B$ is Milnor's $\infty$-universal fibre bundle for $\Gamma$ [7]. Recall that $E$ and $B$ are countable regular CW-complexes. Thus $E \times G$ is regular and, hence, paracompact, since it is the countable union of countable compact subsets, namely $E \times G=\cup_{n} E^{n} \times G$, where $E^{n}$ is the join of $\Gamma$ with itself $(n+1)$ times. Make $\Gamma$ act on the product $E \times G$ via the diagonal map; as in (4.1.1), denote by $E \times_{\Gamma} G$ the decomposition space $(E \times G) / \Gamma$. Consider next the commutative diagram,

$$
\begin{gathered}
G \stackrel{\alpha}{\leftarrow} E \times G \stackrel{\beta}{\rightarrow} E \\
f \downarrow \\
\quad \stackrel{\downarrow}{\leftarrow} E \times{ }_{\Gamma} G \stackrel{\bar{\beta}}{\rightarrow} B,
\end{gathered}
$$

where $\bar{\alpha}$ and $\bar{\beta}$ are induced by the projections $\alpha$ and $\beta$. 
Proposition (5.1.1). The induced cohomology map

$$
\bar{\alpha}^{*}: H^{*}(U) \rightarrow H^{*}\left(E \times{ }_{\Gamma} G\right)
$$

is an isomorphism of algebras.

The proof makes use of the spectral sequence $\left\{E_{r}(\bar{\alpha}), r \geqq 2\right\}$ of the locallytrivial fibration $\bar{\alpha}: E \times_{\Gamma} G \rightarrow U=G / \Gamma$. As is well known $E_{2}=H^{*}\left(B ; \mathfrak{H}^{*}(E)\right)$ $[6, \S 4.17]$. By the local triviality of $\bar{\alpha}$, the paracompactness of $E \times_{\Gamma} G$, and the contractibility of $E$, it follows that $\mathcal{F C}^{q}(E)_{x}=0$ if $q>0$ and $=Z_{p}$ if $q=0$ where $\mathcal{H}^{*}(E)_{x}$ is the stalk of $\mathcal{H}^{*}(E)$ at any $x \in U$. Hence $E_{2}^{*, q}=0$ if $q \neq 0$, and $=H^{*}\left(B, \mathfrak{H C}^{0}(E)\right)$, for $q=0$. Thus we need only show

LEMMA. $\operatorname{HC}^{0}(E)$ is a simple sheaf over $U$.

Proof. Since $\Gamma$ is finite, it is easy to find a compact connected Lie group $G_{\delta}$ and an epimorphism $\phi_{\delta}: G \rightarrow G_{\delta}$ such that $\phi_{\delta} \mid \Gamma$ is an isomorphism. Put $\phi_{\delta}(\Gamma)=\Gamma_{\delta} \subset G_{\delta} . \phi_{\delta}$ induces the commutative diagram,

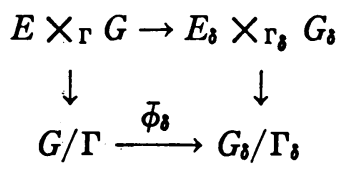

where $E_{\delta}$ is the $\infty$-universal bundle for $\Gamma_{\delta}$. We know that $\mathfrak{H}^{0}\left(E_{\delta}\right)$ is a simple sheaf over $G_{\delta} / \Gamma_{\delta}$. But $\Phi_{\delta}$ induces an isomorphism between the induced sheaf $\Phi_{\delta}^{*} \mathcal{H C}^{0}\left(E_{\delta}\right)$ and $\mathcal{H}^{0}(E)$. Hence the lemma. Q.E.D.

Proposition (5.1.2). The spectral sequence $\left\{E_{r}(\beta), r \geqq 2\right\}$ of the fibration $\bar{\beta}: E \times_{\Gamma} G \rightarrow B$ has the following properties:

(i) the sheaf $\mathrm{H}^{*}(G)$ is isomorphic to the simple sheaf whose stalk is $H^{*}(G)$; and

(ii) $E_{2}(\bar{\beta})=H^{*}(B) \otimes H^{*}(G)$.

(i) is a consequence of the local triviality of $\bar{\beta}$ and the connectedness of $G$, whereas (ii) follows from the fact that $H^{*}(B)$ is finitely generated.

(5.2) Let $G$ be a compact connected group, and let $K$ be a connected, normal, and closed subgroup of $G$. Put $G / K=U$, and let $f$ be the canonical $\operatorname{map} f: G \rightarrow U$.

Proposition (5.2.1). In the spectral sequence $\left\{E_{r}(f), r \geqq 2\right\}$ of the epimorphism $f: G \rightarrow U$, the sheaf of fibres $\mathcal{H}^{*}(K)$ is simple and $E_{2}(f)=H^{*}(U) \otimes H^{*}(K)$.

To prove the proposition, we represent $\mathfrak{F}^{*}(K)$ as a direct limit of simple sheaves. The essential fact is that $f: G \rightarrow U$ is an inverse limit of fibrations of Lie groups by Lie groups (see (4.2.1)). So let $\left\{\left(E_{\alpha}, \phi_{\alpha}, G_{\alpha}\right), \alpha \in J\right\}$ be an inverse system of principal fibre spaces. Denote $E_{\alpha} / G_{\alpha}$ by $B_{\alpha}$ and the natural maps $E_{\alpha} \rightarrow B_{\alpha}$ by $f_{\alpha}$. Assume that, for every $\alpha, E_{\alpha}$ is compact, $B_{\alpha}$ is a complex, and $f_{\alpha}: E_{\alpha} \rightarrow B_{\alpha}$ is locally trivial. Let 


$$
(E, \phi, G)=\underbrace{\lim }_{\alpha}\left(E_{\alpha}, \phi_{\alpha}, G_{\alpha}\right),
$$

and write $B$ and $E / G$ for $\bar{\mu}_{\alpha}$ for the natural map $B \rightarrow B_{\alpha}$ induced by $\mu_{\alpha}: E \rightarrow E_{\alpha}$. Recall that

$$
B=\lim _{\alpha} B_{\alpha},
$$

and that the sheaf $\mathcal{F}^{*}\left(G_{\alpha}\right)$, defined by the cohomology of the fibres of $f_{\alpha}$ over $B_{\alpha}$, is simple; it is naturally isomorphic to the simple sheaf $L_{\alpha}^{\prime}$, whose stalk is $H^{*}\left(G_{\alpha}\right)$. Denote the sheaf $\mathcal{F C}^{*}\left(G_{\alpha}\right)$ by $\mathcal{L}_{\alpha}^{\prime}$, and the sheaf $\bar{\mu}_{\alpha}^{*} \mathcal{L}_{\alpha}^{\prime}$, induced by $\bar{\mu}_{\alpha}$, by $\mathfrak{L}_{\alpha}$. Then the mappings $\mu_{\alpha \beta}: E_{\alpha} \rightarrow E_{\beta}$ induce in a natural way a mapping of sheaves $\hat{\mu}_{\alpha \beta}: \mathfrak{L}_{\alpha} \rightarrow \mathfrak{L}_{\beta}$. Thus,

Proposition (5.2.2). The system $\left\{\mathfrak{L}_{\alpha}, \hat{\mu}_{\alpha \beta}, \alpha, \beta \in J\right\}$ is a direct system of sheaves over $B$.

Observe that each $\mathscr{L}_{\alpha}$ is isomorphic to the simple sheaf $\bar{\mu}_{\alpha}^{*} L_{\alpha}^{\prime}=L_{\alpha}$.

Proposition (5.2.3). The direct limit

$$
\mathscr{L}=\underset{\alpha}{\lim } \mathscr{L}_{\alpha}
$$

is naturally isomorphic to the sheaf $\mathcal{F}^{*}(G)$. In particular, $\mathfrak{F}^{*}(G)$ is simple, with stalk $H^{*}(G)$.

It is not hard to construct a continuous mapping $\mathscr{L} \rightarrow \mathfrak{H}^{*}(G)$ which is an isomorphism on the stalks. Just as easily it can be shown that the direct limit of simple sheaves over a connected space is simple.

(5.3) Suppose now that $f: X \rightarrow Y$ and $f^{\prime}: X^{\prime} \rightarrow Y^{\prime}$ are fibre maps. Then $f \times f^{\prime}: X \times X^{\prime} \rightarrow Y \times Y^{\prime}$ is also a fibre map. Assume that the underlying ring of coefficients is $R$. Consider $E_{r}(f) \otimes E_{r}\left(f^{\prime}\right)$, the tensor product of the spectral sequence of $f$ with the spectral sequence of $f^{\prime}$, and give it the usual product differential and gradation. We wish to define a map $\psi_{r}: E_{r}(f) \otimes E_{r}\left(f^{\prime}\right)$ $\rightarrow E_{r}\left(f \times f^{\prime}\right), r \geqq 2$, of differential graded $R$-modules. We follow the notation of $[6]$.

(5.3.1) First we give a natural map of sheaves

$$
\alpha: \mathscr{L}^{*} \hat{\otimes} \mathcal{L}^{* *} \rightarrow\left(f \times f^{\prime}\right)_{*}\left(\mathfrak{C}^{*}(X ; R) \hat{\otimes} \mathfrak{e}^{*}\left(X^{\prime} ; R\right)\right)=\odot^{*},
$$

where $\mathscr{L}^{*}$ and $\mathscr{L}^{*}$ are $f_{*} \mathfrak{C}^{*}(X ; R)$ and $f_{*}^{\prime} \mathfrak{C}^{*}\left(X^{\prime} ; R\right)$, respectively. Recall $\mathscr{L}^{*} \hat{\otimes} \mathcal{L}^{*}$ is generated by the presheaf which assigns to an open set $U \times V$ of $Y \times Y^{\prime}$ the $R$-module $\mathscr{L}^{*}(U) \otimes_{R} \mathfrak{L}^{* *}(V)=\mathfrak{C}^{*}(X ; R)\left(f^{-1} U\right) \otimes \mathfrak{e}^{*}\left(X^{\prime} ; R\right)\left(f^{\prime-1} V\right)$. The latter module is included in

$$
\begin{aligned}
\left(\mathfrak{e}^{*}(X ; R) \hat{\otimes} \mathfrak{e}^{*}\left(X^{\prime} ; R\right)\right)\left(f \times f^{\prime}\right)^{-1}(U \times V) & \\
& =\left(f \times f^{\prime}\right)_{*}\left(C^{*}(X ; R) \hat{\otimes} \mathfrak{e}^{*}\left(X^{\prime} ; R\right)\right)(U \times V) .
\end{aligned}
$$


Then, by definition, $\alpha$ is the map induced by this inclusion.

(5.3.2) Put $\mathfrak{T}^{*}=\left(f \times f^{\prime}\right)_{*}\left(\mathfrak{C}^{*}\left(X \times X^{\prime} ; R\right)\right)$, and consider the cartesian product map $[6, \S 6]$,

$$
\delta: \mathfrak{C}^{*}(X ; R) \hat{\otimes} \mathfrak{e}^{*}\left(X^{\prime} ; R\right) \rightarrow \mathfrak{e}^{*}\left(X \times X^{\prime} ; R\right) .
$$

Then $f \times f^{\prime}$ induces the map $\left(f \times f^{\prime}\right)_{*}(\delta): \rho^{*} \rightarrow \mathscr{T}^{*}$.

(5.3.3) Let $\mu=\mathfrak{e}^{*}(\alpha) \circ \delta: \mathfrak{C}^{*}\left(Y ; \mathscr{L}^{*}\right) \hat{\otimes} \mathfrak{e}^{*}\left(Y^{\prime} ; \mathfrak{L}^{*}\right) \rightarrow \mathfrak{C}^{*}\left(Y \times Y^{\prime} ; \mathcal{\odot}^{*}\right)$ be the composition of the cartesian product map $\delta[6, \S 6]$ and the map $\mathcal{C}^{*}(\alpha)$ induced by $\alpha$. Then

$$
\lambda=\mathfrak{C}^{*}\left(\left(f \times f^{\prime}\right)_{*}(\delta)\right) \circ \mu: \mathfrak{C}^{*}\left(Y ; \mathfrak{L}^{*}\right) \otimes \mathfrak{C}^{*}\left(Y^{\prime} ; \mathfrak{L}^{* *}\right) \rightarrow \mathfrak{C}^{*}\left(Y \times Y^{\prime} ; \mathfrak{T} \mathbb{T}^{*}\right) .
$$

$\lambda$, on the other hand, induces a map

$$
\psi: C^{*}\left(Y ; \mathscr{L}^{*}\right) \otimes C^{*}\left(Y^{\prime} ; \mathfrak{L}^{* *}\right) \rightarrow C^{*}\left(Y \times Y^{\prime} ; \mathfrak{T}^{*}\right)
$$

of the global sections. Notice that $\psi$ is a map of filtered graded modules. Thus,

Proposition (5.3.4). With the notation as above, $\psi$ induces, for every $r$ such that $2 \leqq r \leqq \infty$,

$$
\psi_{r}\left(f \times f^{\prime}\right): E_{r}(f) \otimes E_{r}\left(f^{\prime}\right) \rightarrow E_{r}\left(f \times f^{\prime}\right),
$$

a homomorphism of differential graded modules. Furthermore, $\psi_{r+1}\left(f \times f^{\prime}\right)$ is induced by $\psi_{r}\left(f \times f^{\prime}\right)$.

Proposition (5.3.5). Suppose that the sheaves $\mathcal{H}^{*}(G ; R)$ and $\mathcal{H}^{*}\left(G^{\prime} ; R\right)$ are simple and $R$ is a field. A ssume further that either $Y$ and $Y^{\prime}$ are compact, or $H^{*}(Y ; R), H^{*}\left(Y^{\prime} ; R\right)$ and $H^{*}\left(Y \times Y^{\prime} ; R\right)$ are finitely generated in every homogeneous dimension. Then $\psi_{r}\left(f \times f^{\prime}\right): E_{r}(f) \otimes E_{r}\left(f^{\prime}\right) \rightarrow E_{r}\left(f \times f^{\prime}\right)$ is an isomorphism of algebras, for all $r \geqq 2$.

First we need

Lemma (5.3.6). The sheaf of coefficients $\mathcal{H}^{*}\left(G \times G^{\prime}\right)$ over $Y \times Y^{\prime}$ is simple whenever $\mathfrak{H C}^{*}(G)$ over $Y$ and $\mathfrak{H C}^{*}\left(G^{\prime}\right)$ over $Y^{\prime}$ are both simple.

The proof of the lemma is not difficult and will be left to the reader. Then the usual universal coefficient theorem implies that $\psi_{2}\left(f \times f^{\prime}\right): E_{2}(f) \otimes E_{2}\left(f^{\prime}\right)$ $\rightarrow E_{2}\left(f \times f^{\prime}\right)$ is an isomorphism of graded vector spaces. Hence $\psi_{r}\left(f \times f^{\prime}\right)$ is also an isomorphism for all $r \geqq 2$. To prove that they are isomorphisms of algebras it is enough to examine the diagram $\left(D_{r}\right)$ following

$$
\begin{aligned}
& E_{r}(f) \otimes E_{r}(f) \otimes E_{r}\left(f^{\prime}\right) \otimes E_{r}\left(f^{\prime}\right) \stackrel{\left[\psi_{r} \otimes \psi_{r}\right] \circ T}{\longrightarrow} E_{r}\left(f \times f^{\prime}\right) \otimes E_{r}\left(f \times f^{\prime}\right) \\
& \begin{array}{ccc}
\psi_{r} \otimes \psi_{r} \downarrow & \psi_{r} \downarrow \\
E_{r}(f \times f) \otimes E_{r}\left(f^{\prime} \times f^{\prime}\right) \longrightarrow & T \circ \psi_{r} & E_{r}\left(f \times f^{\prime} \times f \times f^{\prime}\right)
\end{array} \\
& \begin{aligned}
& d(f) \otimes d\left(f^{\prime}\right) \downarrow \quad \psi_{r} \quad d\left(f \times f^{\prime}\right) \downarrow \\
& E_{r}(f) \otimes E_{r}\left(f^{\prime}\right) \longrightarrow E_{r}\left(f \times f^{\prime}\right)
\end{aligned}
\end{aligned}
$$


where $T$ is the usual "twisting" and $d(f)$ is the map induced by the diagonal $\operatorname{map} f \rightarrow f \times f$.

In $\left(D_{2}\right)$ the upper diagram is commutative because of the commutavity and associativity of the cartesian product. The lower diagram, on the other hand, is commutative because of the naturality of the cartesian product. Since $\left(D_{r}\right)$ is the derived diagram of $\left(D_{r-1}\right)$, the result follows immediately. Q.E.D.

(5.4) Let $f: E \rightarrow B$ be a principal fibre space for the compact connected group. Assume further that either $B$ is compact or $H^{*}\left(B ; Z_{p}\right)$ is finitely generated in every homogeneous dimension. We also assume that $\mathcal{H}^{*}(G)$ is simple over $B$. Thus $E_{2}^{* * *}(f)=H^{*}\left(B ; Z_{p}\right) \otimes H^{*}\left(G ; Z_{p}\right)$. Since $G$ acts freely on $E$, we get the commutative diagram

$$
\begin{aligned}
E \times G & \rightarrow \\
f \times f^{\prime} \downarrow & \\
B \times\{b\} & \rightarrow B,
\end{aligned}
$$

where $f^{\prime}$ is the constant map. This induces a homomorphism of algebras, $\phi_{r}: E_{r}(f) \rightarrow E(f) \otimes H^{*}(G)$ by (5.3.5). Observe that $\phi_{2}$ restricted to the fibre $H^{*}(G)$ is merely the diagonal map in the Hopf-algebra $H^{*}(G)$.

Definition (5.4.1). Let $u \in H_{*}(G ; R)=\operatorname{Hom}\left(H^{*}(G ; R) ; R\right)$. Put $\vartheta_{u}$ $=(1 \otimes u) \circ \phi_{r}: E_{r}(f) \rightarrow E_{r}(f) . \vartheta_{u}$ is called the Leray operator corresponding to $u$.

The following two propositions sum up the main properties of these operators.

Proposition (5.4.2). For all $r \geqq 2$ and $u \in H_{*}(G)$ :

(i) $\vartheta_{u}$ is a homomorphism of vector spaces;

(ii) $\vartheta_{u}: E_{r}^{p, q} \rightarrow E_{r}^{p, q-l}$, where $u \in H_{l}(G)$;

(iii) $\vartheta_{u} \circ d_{r}=d_{r} \circ \vartheta_{u}$; $E_{r+1}$

(iv) $\vartheta_{u} \circ \kappa_{\tau+1}^{r}=\kappa_{\tau+1}^{r} \circ \vartheta_{u}$, where $\kappa_{\tau+1}^{r}$ is the map taking the cocycles of $E_{r}$ in

(v) let $b \otimes g \in E_{2}^{*, *}(f)=H^{*}(B) \otimes H^{*}(G)$; then if $u \in H_{*}(G)$, it follows that $\vartheta_{u}(b \otimes g)=b \otimes \vartheta_{u}(g)$.

The proofs are very simple.

Next we shall prove the following fundamental proposition.

Proposition (5.4.3). Consider the spectral sequence $\left\{E_{r}(f), r \geqq 2\right\}$ of the principal fibration $f: E \rightarrow B$. Assume that $E_{2}(f)=\cdots=E_{8}(f)$. Then if $d_{s}: E_{s}^{0,8-1} \rightarrow E_{s}^{s, 0}$ is trivial, it follows that the differential operator $d_{s}$ is trivial and, hence, $E_{s}(f)=E_{s+1}(f)$.

Proof. Since $E_{s}(f)=E_{2}(f)=H^{*}(G) \otimes H^{*}(G)$ and $d_{s}$ is a derivation, it is enough to prove that $d_{\text {s }}$ restricted to $H^{*}(G)$ is trivial. So take $z \in H^{n}(G)$. Then

$$
d_{s}(z)=\sum_{j} b_{j} \otimes g_{j},
$$


with $b_{j} \in H^{s}(B)$ and $g_{j} \in H^{n-8+1}(G)$; we can assume that the $g_{j}$ 's are linearly independent over $Z_{p}$. Complete the set $\left\{g_{j}\right\}$ to a basis $S$ of $H^{*}(G)$. Choose some $u_{i} \in H_{n-i+1}(G)$ such that $u_{i}\left(g_{i}\right)=1$ and $u_{i}(x)=0$ for all $x \neq g_{i} \in S$. Then by (5.4.2) we get

$$
\vartheta_{u_{i}}\left(d_{s}(z)\right)=\sum_{j} b_{j} \otimes \vartheta_{u_{i}}\left(g_{j}\right)
$$

But $\vartheta_{u_{i}}\left(g_{j}\right)=0$ if $g_{j} \neq g_{i}$. To see this, let $\phi_{s}\left(g_{j}\right)=g_{j} \otimes 1+1 \otimes g_{j}+\sum_{k} g_{j_{k}}^{\prime} \otimes g_{j_{k}}^{\prime \prime}$, where $\operatorname{deg} g_{j_{k}}^{\prime}, \operatorname{deg} g_{j_{k}}^{\prime}<\operatorname{deg} g_{j}$. Hence $\vartheta_{u_{i}}\left(g_{j}\right)=1$ if $j=i$, and $=0$ if $j \neq i$. Thus

$$
d_{s}\left(\left(\vartheta_{u_{i}}\right)(z)\right)=\vartheta_{u_{i}}\left(d_{s} z\right)=b_{i}
$$

But $\vartheta_{u_{i}}(z) \in H^{s-1}(G)$; therefore $d_{s}\left(\vartheta_{u_{i}}(z)\right)=b_{i}=0$. We can repeat this procedure for every element $b_{i}$ appearing in the sum $\left({ }^{*}\right)$. Thus $d_{s}(z)=0$. Q.E.D.

6. Proof of Theorem (2.2.1). We shall assume here that the domain of coefficients for cohomology is $Z_{p}$, the prime field of characteristic $p$. Let $G$ be a compact connected group and $\Gamma$ a subgroup isomorphic to $Z_{p^{n}}$, where $n$ is some integer greater than, or equal to, 1 . Consider the natural map $f: G \rightarrow U$ $=G / \Gamma$ and the space $E \times_{\Gamma} G$ which it defines (see (5.1)). There are two naturally defined locally trivial fibrations: $\bar{\alpha}: E \times_{\Gamma} G \rightarrow U$ and $\bar{\beta}: E \times_{\Gamma} G \rightarrow B$. Proposition (5.1.1) implies that $\bar{\alpha}^{*}: H^{*}(U) \rightarrow H^{*}\left(E \times_{\Gamma} G\right)$ is an isomorphism of the Cech cohomology algebras. Moreover, by Proposition (5.1.2), we know that in the spectral sequence $\left\{E_{r}(\bar{\beta}), r \geqq 2\right\}$,

$$
E_{2}(\bar{\beta})=H^{*}(B) \otimes H^{*}(G) \text {. }
$$

It is understood, of course, that an arbitrary imbedding $i: G \rightarrow E \times_{\Gamma} G$ of $G$ as a fibre has been chosen and that the sheaf $\mathrm{H}^{*}(G)$ is identified with $H^{*}(G)$ by means of this imbedding. As is well known, $f^{*}=i^{*} \circ \bar{\alpha}^{*}$ and $\bar{\alpha}^{*-1} \circ \bar{\beta}^{*}=\chi^{*}$, where $\chi$ is that mapping of $U$ in $B$ inducing the fibration $f: G \rightarrow U$. Thus, the proof of Theorem (2.2.1) is reduced to the analysis of the sequence $\left\{E_{r}(\bar{\beta}), r \geqq 2\right\}$. We shall do that for the case $p>2$, the other case being similar. The proof is a consequence of the following lemmas.

LEMма (6.1). $\operatorname{ch}(f) \approx E(x, 1) \otimes P(y, 2) /\left(y^{k}\right)$, where $k$ is some positive integer.

Proof. Put $\bar{x}=\chi^{*}(x)$ and $\bar{y}=\chi^{*}(y)$ and observe first of all that the element $\bar{y}$ of $H^{*}(G / \Gamma)$ is nilpotent because $G / \Gamma$ is compact. Thus there exists a Lie group $G^{\prime}$ and an epimorphism $\phi: G \rightarrow G^{\prime}$ such that (i) $\phi$ restricted to $\Gamma$ is an isomorphism and (ii) $\phi^{*}$ maps the characteristic ring of the fibrations $G^{\prime} \rightarrow G^{\prime} / \phi(\Gamma)$ isomorphically onto the characteristic ring of the fibration $G \rightarrow G / \Gamma$. Thus, we need prove Lemma (6.1) only for the case when $G$ is a Lie group. So we assume that $G$ is a Lie group and choose a circle subgroup $S$ of $G$ containing $\Gamma$. Consider a universal bundle $\lambda: E_{G \rightarrow B}$ for $G$. Since $\Gamma$ is a subgroup of $G$, we obtain in a well-known manner the following commutative diagram: 


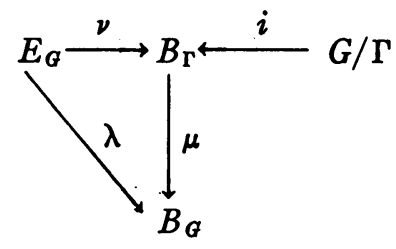

where $B_{\Gamma}=E_{G} / \Gamma, \mu$ and $\nu$ are fibrations, and $i$ is the inclusion map of the fibre $G / \Gamma$. Observe that $i$ can be taken as the classifying map for the fibration $f: G \rightarrow G / \Gamma$. Hence im $i^{*}=\operatorname{ch}(f)$. Similarly, the inclusion $S \subset G$ induces the commutative diagram

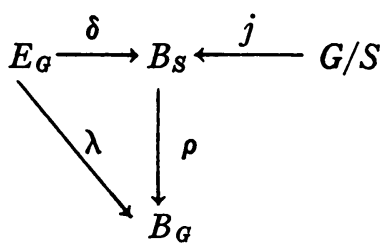

Again $j$ can be taken as the classifying map for $h: G \rightarrow G / S$, and thus im $j^{*}$ is equal to the characteristic algebra of $h$; that is, im $j^{*}=\operatorname{ch}(h)$. Now since $\Gamma$ is a subgroup of $S, \mu$ factors through $B_{S}$, and we get the commutative diagram:

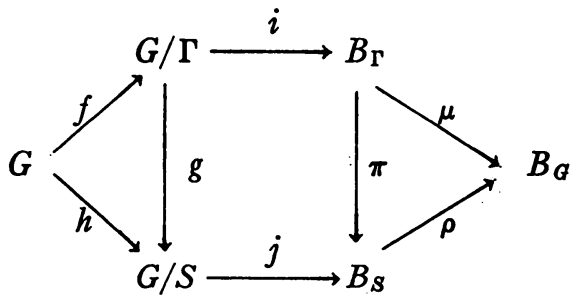

Consider the fibration $\pi: B_{\Gamma} \rightarrow B_{S}$, whose fibre is $S / \Gamma$. Observe that $S / \Gamma$ is totally nonhomologous to zero $\bmod p$ in $B_{\Gamma}$. By naturality, $S / \Gamma$ is also totally nonhomologous to zero $\bmod p$ in $G / \Gamma$. Hence the spectral sequence $\left\{E_{r}(g), r \geqq 2\right\}$, of the fibration $g$ is trivial; i.e.,

$$
H^{*}(G / S) \otimes H^{*}(S / \Gamma)=E_{2}(g)=E_{\infty}(g) .
$$

To finish the proof, observe that $\operatorname{ch}(h) \approx P\left(y^{\prime}, 2\right) /\left(y^{\prime k}\right)$, where $y^{\prime}=j^{*}(y)$. Thus, in $H^{*}(G / \Gamma), \bar{y}^{k}=0$ but $\bar{y}^{k-1} \neq 0$. Also $\bar{x} \bar{y}^{k-1} \neq 0$ in $H^{*}(G / \Gamma)$, since it is so in $E_{2}(g)$, and $E_{2}(g)=E_{\infty}(g)=E_{0}\left(H^{*}(G / \Gamma)\right)$. Q.E.D.

Lemma (6.2). Suppose $\Gamma$ is invariant in $G$. Then $\operatorname{ch}(f)$ is a sub-Hopfalgebra of $H^{*}(U)$, primitively generated by $\chi^{*}(x)$ and $\chi^{*}(y)$. Moreover, $\chi^{*}(y)$ has height $p^{*}$ for some integer $s \geqq 0$, and ker $\chi^{*}$ is the ideal of $H^{*}(B)$ generated by $y^{p}$. 
Proof. Since $\Gamma$ is abelian, the mapping $\phi: \Gamma \times \Gamma \rightarrow \Gamma$ defining the multiplication on $\Gamma$ is a homomorphism. Hence $\phi$ induces $H$-structures on $E$ and $B$ with respect to which $\gamma$ is a homomorphism of $H$-spaces. Observe that $\Gamma$ is central in $G$, since it is invariant and discrete. Hence the $H$-structure on $E$, together with the multiplication on $G$, induces an $H$-structure on $E \times_{\Gamma} G$, and the mappings $\bar{\alpha}$ and $\bar{\beta}$ are homomorphisms of $H$-spaces. Thus $H^{*}\left(E \times_{\Gamma} G\right)$ and $H^{*}(B)$ are Hopf-algebras, and $\bar{\alpha}^{*}$ and $\bar{\beta}^{*}$ are homomorphisms of Hopfalgebras. Recall that $x$ and $y$ are primitive elements of $H^{*}(B)$. Hence $\chi^{*}(x)$ and $\chi^{*}(y)$ are primitive elements of $H^{*}(U)$, since $\bar{\alpha}^{*}$ and $\bar{\beta}^{*}$ are homomorphisms of Hopf-algebras. The rest follows from standard facts on Hopfalgebras. Q.E.D.

(6.1) and (6.2) imply the first part of the theorem.

Lemma (6.3). Suppose $k$ is the integer defined in Lemma (6.1). Then $d_{2 k}$ is the first nontrivial differential operator of $\left\{E_{r}(\bar{\beta}), r \geqq 2\right\}$ and is an epimorphism of $H^{2 k-1}(G)=E_{2 k}^{0,2 k-1}$ onto $H^{2 k}(B)=E_{2 k}^{2 k, 0}$. Hence there exists an element $b$ in $H^{2 k-1}(G)$ such that $d_{2 k}(b)=y^{k}$.

Proof. If $k=1$, then $\chi^{*}(y)=0$, and the lemma is true. So assume $k>1$. By (6.1), $d_{2}: E_{2}^{0,1} \rightarrow 0 \in E_{2}^{2,0}$. Proposition (5.4.3) implies $d_{2}=0$. A simple induction argument completes the proof of the first part. The second part is clear. Q.E.D.

Lemma (6.4). $H^{*}(G)=L \otimes\{b\}$, where $\{b\}$ is the module generated by $b$, and $L$ is the part of the kernel of $d_{2 k}$ that lies in $H^{*}(G)$.

Proof. Take an element $x \in H^{*}(G)$. Then $d_{2 k}(x)=y^{k} \otimes x^{\prime}$, where $x^{\prime} \in H^{*}(G)$. Now, since $0=d_{2 k}^{2}(x)=y^{k} d_{2 k}\left(x^{\prime}\right)$, and since multiplication by $y^{k}$ is a monomorphism of $E_{2 k}$, it follows that $d_{2 k}\left(x^{\prime}\right)=0$. Therefore $x-b x^{\prime} \in L$, and, hence, $H^{*}(G)$ is generated by $L$ and $b$. To prove $H^{*}(G)=L \otimes\{b\}$, take $x \in L$. Then $d_{2 k}(x b)=y^{k} \otimes x$, and, therefore, $x b=0$ if and only if $x=0$. Q.E.D.

Notice that $b$ has odd degree when $p>2$. Hence $b^{2}=0$. If $p=2$, degree $b$ need neither be odd nor $b^{2}=0$. We can say only that $b^{2} \in L$, for $d_{2 k}\left(b^{2}\right)$ $=2 b d_{2 k}(b)=0$.

Lemma (6.5). $E_{2 k+1}(\bar{\beta})=\left(E(x, 1) \otimes P(y, 2) /\left(y^{k}\right)\right) \otimes L$, where $L$ is as in (6.4).

Proof. By (6.4), the group of coboundaries of $E_{2 k}$ is the ideal generated by $b$. Therefore it is enough to show that the cocycles are the elements of $(E(x, 1) \otimes P(y, 2)) \otimes L^{*}$. The elements of the latter group are clearly cocycles. To prove that they are all the cocycles there are, take $z \in E_{2 k}$. Then $z=y^{i} \otimes z^{\prime}$, or $x y^{i} \otimes z^{\prime}$, where $z^{\prime} \in H^{*}(G)$. Assume $z$ is a cocycle. If $z=x y^{i} \otimes z^{\prime}$, then $0=d_{2 k}(z)$ $=x d_{2 k}\left(y^{i} \otimes z^{\prime}\right)$; but $d_{2 k}\left(y^{i} \otimes z^{\prime}\right) \in E_{2 k}^{2 k+2 i, *}$ and multiplication by $x$ is a monomorphism of $E_{2 k}^{2 k+2 i, *}$ in $E_{2 k}^{2 k+2 i+1, *}$. Hence $d_{2 k}\left(y^{i} \otimes z^{\prime}\right)=0$; thus we may just as well assume that $z=y^{i} \otimes z^{\prime}$. In this case a similar argument shows that $z$ is a cocycle if and only if $z^{\prime} \in L$. Hence $d_{2 k}(z)=0$ implies that $z \in E(x, 1) \otimes P(y, 2)$ $\otimes L$. Q.E.D. 
Let us observe that $\operatorname{im} i^{*} \subset L$. We shall show

LEMMA (6.6). im $i^{*}=L$.

Proof. It is enough to prove that

$$
\text { for all } x \in L, \quad d_{r}(x)=0, \quad \text { for } r \geqq 2 .
$$

Since the first nontrivial differential operator is $d_{2 k},\left(^{*}\right)$ holds when $2 \leqq r$ $\leqq 2 k-1$. By definition, $d_{2 k}=0$ on $L$. Finally, Lemma (6.5) shows that no nontrivial elements exist when the base degree is greater than or equal to $2 k$. Thus (*) holds also when $r \geqq 2 k+1$. Q.E.D.

Hence $E_{\infty}(\bar{\beta})=\left(E(x, 1) \otimes P(y, 2) /\left(y^{k}\right)\right)^{*} \otimes$ im $i^{*}$. This implies that $H^{*}(U) / / \operatorname{ch}(f)=\operatorname{im} f^{*}$. Finally, by $(6.4), H^{*}(G) / / \operatorname{im~} f^{*}=E(b, 2 k-1)$, where $E(\bar{b}, 2 k-1)$ is the exterior algebra on the element $\bar{b}$ whose degree is $2 k-1$. Notice that $b^{2}=0$. This finishes the proof of Theorem (2.2.1).

7. Proof of Theorem (2.3.1). Let the notation be as in (2.3), and suppose

(7.1) Case 1. $\Gamma=Z_{p}$.

Since $\Gamma$ is invariant in $G, \operatorname{ch}(f)$ is a sub-Hopf-algebra of $H^{*}(U)$, as follows from (6.2). By Theorem (2.2.1) we get the following exact sequence of Hopfalgebras:

$$
\left(\mathrm{S}_{1}\right): \quad 1 \rightarrow \operatorname{ch}(f) \rightarrow H^{*}(U) \stackrel{f^{*}}{\rightarrow} H^{*}(G) \rightarrow E(\bar{b}, 2 k-1) \rightarrow 1 .
$$

Now $\operatorname{im} f^{*}$ is a sub-Hopf-algebra of $H^{*}(G)$; hence $\left(\mathrm{S}_{1}\right)$ is equivalent to the following two exact sequences of Hopf-algebras and homomorphisms:

$$
\begin{aligned}
& \left(\mathrm{S}_{1}^{\prime}\right): 1 \rightarrow \operatorname{ch}(f) \rightarrow H^{*}(U) \stackrel{f^{*}}{\rightarrow} \operatorname{im} f^{*} \rightarrow 1, \\
& \left(\mathrm{~S}_{1}^{\prime \prime}\right): 1 \rightarrow \operatorname{im} f^{*} \rightarrow H^{*}(G) \rightarrow E(b, 2 k-1) \rightarrow 1 .
\end{aligned}
$$

Let us write $V_{f}$ and $L_{f}$ for the spaces of primitive elements and indecomposable elements, respectively, of im $f^{*}$. Similarly, let $V_{x}$ and $L_{x}$ stand for the corresponding spaces of $\operatorname{ch}(f)$. Now we shall proceed to examine $\left(S_{1}^{\prime}\right)$ and $\left(\mathrm{S}_{1}^{\prime \prime}\right)$.

Lemma (7.1.1) $\operatorname{ch}(f)=P(y, 1) /\left(y^{2}\right)$, when $p=2$, and $=E(x, 1)$, when $p>2$.

Proof. ( $\left.\mathrm{S}_{1}^{\prime}\right)$ induces the following sequence of primitive elements [8]:

$$
0 \rightarrow V_{\chi} \rightarrow V_{u} \rightarrow V_{f}
$$

By assumption, $V_{u}$ contains no nontrivial elements of even degree; therefore, the same is true of $V_{\chi}$. This is enough to imply the lemma; for, if $p=2, V_{::}$ is generated by the 2-powers of $y, y, y^{2}, \cdots$, which are all, except for $y$, of even degree and, hence, $y^{2}=0$. Similarly for $p>2$. Q.E.D.

By assumption, $H^{*}(U)$ is generated by elements of odd degree and height 2. Hence the same is true of im $f^{*}$. So the Samelson-Leray Theorem [8, Theorem (4.10)] implies that 
LEMMa (7.1.2). The natural mapping of $V_{f} \rightarrow L_{f}$ is an isomorphism.

Thus, by (7.1.2), $\left(\mathrm{S}_{1}^{\prime \prime}\right)$ states that $H^{*}(G)$ is an extension of an exterior algebra by an exterior algebra. This is a situation we examined in (3.7.1). Therefore we conclude the first part of (2.3.1):

(7.1.3) The natural mapping $V_{o} \rightarrow H^{*}(G)$ extends to an isomorphism $\sigma_{\theta}: E\left(V_{\vartheta}\right) \rightarrow H^{*}(G)$ of Hopf-algebras. Moreover, the sequence of primitive spaces,

$$
0 \rightarrow V_{f} \rightarrow V_{o} \rightarrow\{b\} \rightarrow 0,
$$

is exact.

Similarly, by (7.1.1) and (7.1.2), we conclude that

(7.1.4) The sequence of primitive spaces,

$$
0 \rightarrow\{x\} \rightarrow V_{u} \stackrel{\hat{f}}{\rightarrow} V_{f} \rightarrow 0,
$$

induced by $\left(\mathrm{S}_{1}^{\prime}\right)$, is exact.

Now, to finish the proof of (2.3.1) in the case when $\Gamma=Z_{p}$, notice that (7.1.4) implies that ker $\hat{f} \subset V_{u}^{1}$, the subspace consisting of elements of degree 1 , while (7.1.3) implies that coker $\hat{f}=V_{o} / V_{f}$ consists of elements of degree 1 . Both imply that rank $V_{o}=$ rank $V_{u}$.

(7.2) Case 2. $\Gamma$ is a finite abelian group.

In this case the epimorphism $f: G \rightarrow U$ can be factored into "cyclic" epimorphisms; i.e., there is a finite chain of compact connected groups,

$$
G=G_{0} \stackrel{f_{0}}{\rightarrow} G_{1} \stackrel{f_{1}}{\rightarrow} G_{2} \rightarrow \cdots \stackrel{f_{n}}{\rightarrow} G_{n}=U,
$$

such that $\Gamma_{i}=\operatorname{ker} f_{i}$, for all $i$, is cyclic of prime order, and $f=f_{n} \circ \cdots \circ f_{1} \circ f_{0}$. If the order of $\Gamma_{i}$ is a prime, $q \neq p$, then $f_{i}^{*}$ is an isomorphism. If it is $p$, apply Case 1.

(7.3) Case 3. $\Gamma$ is totally disconnected.

The argument is similar to that of Case 2 : factor $f$ into an inverse system of "finite" epimorphisms. Q.E.D.

8. Proof of Theorem (2.4.1). Let

$$
\text { (S) : } 1 \rightarrow K \stackrel{i}{\rightarrow} G \stackrel{f}{\rightarrow} U \rightarrow 1
$$

be an exact sequence of compact connected groups and homomorphisms. By the results of $\S 4, f$ is a principal fibre map. By Proposition (5.2.1), the sheaf $\mathfrak{F}^{*}(K)$ is simple and

$$
E_{2}(f)=H^{*}(U) \otimes H^{*}(K) .
$$

(i) Suppose that $i^{*}: H^{*}(G) \rightarrow H^{*}(K)$ is an epimorphism. Then the differential operators $d_{2}, d_{3}, \cdots$ of $\left\{E_{r}(f), r \geqq 2\right\}$ are all trivial. Hence, 


$$
E_{\infty}=E_{2}=H^{*}(U) \otimes H^{*}(K) .
$$

Thus $f^{*}$ is a monomorphism, and $H^{*}(K)=H^{*}(G) / / f^{*} H^{*}(U)$.

(ii) Conversely, assume $f^{*}: H^{*}(U) \rightarrow H^{*}(G)$ is a monomorphism. Then

$$
d_{2}: H^{1}(K) \equiv E_{2}^{0,1} \rightarrow H^{2}(U)=E_{2}^{2,0}
$$

is the zero homomorphism. Thus, by Proposition (5.4.3), $d_{2}=0$ on all of $E_{2}$, and $E_{2}=E_{3}$.

Now, by an obvious induction, $E_{\infty}=E_{2}=H^{*}(U) \otimes H^{*}(K)$. Hence, $i^{*}$ is an epimorphism, and $H^{*}(K)=H^{*}(G) / / f^{*} H^{*}(U)$. Q.E.D.

9. Proof of Theorem (2.4.2). Let

$$
\begin{aligned}
& 1 \rightarrow K^{\prime} \stackrel{i^{\prime}}{\rightarrow} G^{\prime} \stackrel{f^{\prime}}{\rightarrow} U^{\prime} \rightarrow 1 \\
& \text { C: } \quad \downarrow \phi_{k} \downarrow \phi_{g} \downarrow \phi_{u} \\
& 1 \rightarrow K \stackrel{i}{\rightarrow} G \stackrel{f}{\rightarrow} U \rightarrow 1
\end{aligned}
$$

be a commutative diagram of compact connected groups and homomorphisms; the rows are exact, while the vertical maps are epimorphisms with totally disconnected kernels. Let us consider the cohomology diagram $C^{*}$ induced by $\mathrm{C}$ :

$$
\begin{aligned}
& 1 \longrightarrow H^{*}\left(U^{\prime}\right) \stackrel{f^{\prime *}}{\longrightarrow} H^{*}\left(G^{\prime}\right) \stackrel{i^{\prime *}}{\longrightarrow} H^{*}\left(K^{\prime}\right) \longrightarrow 1 \\
& \mathrm{C}^{*}: \quad \begin{array}{c}
\uparrow \phi_{u}^{*} \\
\end{array} \stackrel{f^{*}}{\longrightarrow} \stackrel{\uparrow \phi_{0}^{*}}{\longrightarrow} H^{*}(G) \stackrel{i^{*}}{\longrightarrow} \stackrel{\uparrow}{\longrightarrow} H^{*}(K) \longrightarrow 1 .
\end{aligned}
$$

In order to prove (2.4.2) we shall assume the lower (upper) row of $\mathrm{C}^{*}$ is exact and then investigate when the upper (lower) row is also exact. First we prove

Proposition (9.1). Suppose that $\operatorname{ker} \phi_{0} \cap K^{\prime}=\{1\}$; if $f^{*}$ is a monomorphism, then $f^{*}$ is also a monomorphism.

The proof is a simple application of Theorem (2.4.1).

Assume now that

$$
i^{\prime}: \operatorname{ker} \phi_{k} \approx \operatorname{ker} \phi_{o}=Z_{p}
$$

Thus $\operatorname{ker} \phi_{u}=1$, or, equivalently, $\phi_{u}=$ identity. It is easy to see that the left part of diagram $\mathrm{C}$ leads to the following commutative diagram:

$$
\begin{array}{rr}
H^{*}(G) \stackrel{i^{*}}{\rightarrow} H^{*}(K) \\
\chi_{0}^{*} \uparrow & \uparrow \chi_{k}^{*} \\
H^{*}(B) & \leftarrow: H^{*}(B)
\end{array}
$$


where $B$ is the base space of the classifying bundle of $Z_{p}$ (see (4.1.1)) and $\chi_{k}^{*}$ and $\chi_{0}^{*}$ are the characteristic maps of $\phi_{k}$ and $\phi_{0}$, respectively. Thus $i^{*}$ maps $\operatorname{ch}\left(\phi_{0}\right)$ on to $\operatorname{ch}\left(\phi_{k}\right)$. For notational convenience, let $x_{0}=\chi_{\theta}^{*}(x), y_{0}=\chi_{0}^{*}(y)$, and let $x_{k}$ and $y_{k}$ have similar meanings. Then, by Theorem (2.2.1),

$$
\begin{aligned}
\operatorname{ch}\left(\phi_{0}\right) & =P\left(y_{0}, 1\right) /\left(y^{l_{0}}\right), \quad \text { if } p=2, \text { and } \\
& =E\left(x_{0}, 1\right) \otimes P\left(y_{0}, 2\right) /\left(y^{l}\right), \quad \text { if } p>2,
\end{aligned}
$$

while

$$
\begin{aligned}
\operatorname{ch}\left(\phi_{k}\right) & =P\left(y_{k}, 1\right) /\left(y^{l_{k}}\right), \quad \text { if } p=2, \text { and } \\
& =E\left(x_{k}, 1\right) \otimes P\left(y_{k}, 2\right) /\left(y^{l_{k}}\right), \quad \text { if } p>2 .
\end{aligned}
$$

Moreover, $l_{g}$ and $l_{k}$ are $p$-powers. For notational convenience, put $s=l_{k}$ if $p=2$, and $s=2 l_{k}$ if $p>2$ : and put $t=l_{g}$ if $p=2$, and $t=2 l_{g}$ if $p>2$. Observe that $s \leqq t$, since $i^{*}$ maps $\operatorname{ch}\left(\phi_{0}\right)$ onto $\operatorname{ch}\left(\phi_{k}\right)$. The result we seek is

Proposition (9.2). Let the notation be as in the previous paragraph. Suppose that $i^{*}$ is an epimorphism and $\operatorname{ker} \phi_{k} \approx \operatorname{ker} \phi_{o}=Z_{p}$ :

(i) $\operatorname{ker} f^{*}=(z) \subset H^{*}\left(U^{\prime}\right)=H^{*}(U)$, where $(z)$ is the ideal generated by the element $z$ which satisfies the relation $f^{*}(z)=y_{0}^{l_{k}} \in H^{*}(G)$;

(ii) $\operatorname{ker} i^{*}$ is the ideal generated by an element $b \in H^{t-1}\left(G^{\prime}\right)$ and the elements of positive degree in im $f^{*}$;

(iii) $\operatorname{im} i^{* *}=\operatorname{im} \phi_{k}^{*}$.

Corollary (9.2.1). Consider the diagram C, and suppose $i^{*}$ is an epimorphism or, equivalently, $f^{*}$ is a monomorphism. Then if $H^{*}(U)$ is generated by elements of odd degree and height 2 and $i: \operatorname{ker} \phi_{k} \rightarrow \operatorname{ker} \phi_{0}$ is an isomorphism, it follows that $f^{*}$ is also a monomorphism. In particular, $H^{*}\left(G^{\prime}\right) / / f^{\prime *} H^{*}\left(U^{\prime}\right)$ $=H^{*}\left(K^{\prime}\right)$ as an algebra.

Proof. (Case 1) $\operatorname{ker} \phi_{k} \approx \operatorname{ker} \phi_{o}=Z_{p}$.

Since $l_{k}$ is a power of $p$, the fact that $y_{0}$ is a primitive element in $H^{*}(G)$ implies that $y_{0}^{l_{k}}$ is also primitive. As $f^{*}$ is a monomorphism, (9.2) implies that $f^{*-1} y_{0}^{l_{k}}=z \in H^{*}(U)$ is also primitive. Observe that deg $z$ is even. But the Samelson-Leray Theorem implies that $H^{*}(U)$ has no nonzero primitive elements of even degree. Hence $z=0$, and thus $\operatorname{ker} f^{* *}=(z)=0$; i.e., $f^{\prime *}$ is a monomorphism. The rest follows by Theorem (2.4.1).

(Cases 2 and 3) These are when $\operatorname{ker} \phi_{k}=\operatorname{ker} \phi_{g}=\mathrm{a}$ finite group and a totally disconnected group, respectively; they can be reduced to Case 1 (see §7). Q.E.D.

Let us assume Proposition (9.2) for the moment and use it for the

Proof of Theorem (2.4.2). Let

$$
1 \rightarrow K \stackrel{i}{\rightarrow} G \stackrel{f}{\rightarrow} U \rightarrow 1
$$

be an exact sequence of compact connected groups and homomorphisms. Con- 
sider the following diagram of compact connected groups and homomorphisms:

$$
\begin{array}{ll}
1 \rightarrow & \hat{K} \stackrel{\hat{\imath}}{\rightarrow} \hat{G} \stackrel{\hat{f}}{\rightarrow} \hat{G} / \hat{K} \rightarrow 1 \\
(\mathrm{~A}): \begin{array}{lll} 
& \downarrow \phi_{k} \quad \downarrow \phi_{o} \quad \downarrow \phi_{u}{ }^{\prime}
\end{array} \\
1 \rightarrow & K \stackrel{i}{\rightarrow} G \stackrel{f}{\rightarrow} U \longrightarrow 1,
\end{array}
$$

where $\hat{K}$ and $\hat{G}$ are the canonical groups associated with $K$ and $G$, respectively, while $\phi_{k}$ and $\phi_{g}$ are the homomorphisms that go with them (see (3.4)); $\phi_{u}{ }^{\prime}$, on the other hand, is induced by $\phi_{g}$. Recall that $\hat{G}=\prod_{i} G_{i} \times G^{c}$, where each $G_{i}$ is a simply connected and compact Lie group and $G^{c}$ is the connected component of the identity of the center of $G$. Put $D_{i}=$ the projection of $\operatorname{ker} \phi_{0}$ on $G_{i}$ and $D^{c}=$ the projection of $\operatorname{ker} \phi_{g}$ on $G^{c}$. Finally, form $\prod_{i} D_{i} \times D^{c}$, and denote it by $D$. Consider now a new diagram, $\left(\mathrm{A}^{*}\right)$ say, induced by $(\mathrm{A})$ :

$$
\begin{aligned}
& 1 \rightarrow K \stackrel{i}{\rightarrow} G \stackrel{f}{\longrightarrow} U \longrightarrow 1 \\
& \left(\mathrm{~A}^{*}\right): \quad \downarrow \rho_{k} \quad \downarrow \rho_{o} \quad \downarrow \rho_{u} \\
& 1 \rightarrow \check{K} \rightarrow \check{G} \rightarrow \check{G} / \check{K} \rightarrow 1 \text {, }
\end{aligned}
$$

where $\check{G}=\hat{G} / D, \rho_{0}$ is the map induced by the natural projection $\hat{G} \rightarrow \hat{G} / D$, $\check{K}=\operatorname{im}\left(\rho_{g} \circ i\right)$, and $\rho_{u}$ is the map induced by $\rho_{g} .\left(A^{*}\right)$, in its turn, induces

$$
\begin{aligned}
& 1 \rightarrow K \stackrel{i}{\longrightarrow} G \stackrel{f}{\longrightarrow} U \longrightarrow 1 \\
& \downarrow \rho_{k} \quad \downarrow \quad \downarrow \| \\
& \left(\mathrm{A}^{* *}\right): 1 \rightarrow \check{K} \rightarrow G / \Lambda_{k} \longrightarrow U \longrightarrow 1 \\
& \downarrow \| \quad \downarrow \quad \downarrow \rho_{u} \\
& 1 \rightarrow \check{K} \longrightarrow \check{G} \longrightarrow \check{G} / \hat{K} \rightarrow 1,
\end{aligned}
$$

where $\Lambda_{k}=\operatorname{ker} \rho_{k}$. The third row induces the following exact sequence of Hopf-algebras and homomorphisms:

$$
\left(\mathrm{S}_{3}\right): 1 \leftarrow H^{*}(\check{K}) \leftarrow H^{*}(\check{G}) \leftarrow H^{*}(\check{G} / \check{K}) \leftarrow 1 .
$$

Proposition (9.1) implies that the sequence

$$
\left(\mathrm{S}_{2}\right): 1 \leftarrow H^{*}(\check{K}) \leftarrow H^{*}\left(G / \Lambda_{k}\right) \leftarrow H^{*}(U) \leftarrow 1
$$

is exact; and, finally, Corollary (9.2.1) implies that the desired sequence

$$
1 \leftarrow H^{*}(K) \stackrel{i^{*}}{\leftarrow} H^{*}(G) \stackrel{f^{*}}{\leftarrow} H^{*}(U) \leftarrow 1
$$

is exact. Q.E.D.

(9.3) We shall give now the proof of Proposition (9.2) in the form of lemmas. 
Lemma (9.3.1). There is an element $z$ in $H^{*}(U)$ such that $f^{*}(z)=y_{0}^{l_{k}}$.

Proof. Since $i^{*}$ maps $\operatorname{ch}\left(\phi_{0}\right)$ onto $\operatorname{ch}\left(\phi_{k}\right)$ taking $x_{0}$ to $x_{k}$ and $y_{0}$ to $y_{k}$, it follows that $i^{*}\left(y_{0}^{l_{k}}\right)=0$ in $H^{*}(K)$. But $l_{0}$ is a power of $p$, and $y_{0}$ is primitive. Thus, by the exactness of the sequence of primitive elements $[8$, Theorem (3.6)],

$$
0 \rightarrow V_{u} \stackrel{f^{*}}{\rightarrow} V_{o} \stackrel{i^{*}}{\rightarrow} V_{k}
$$

we get a primitive element $z$ in $H^{*}(U)$ such that $f^{*}(z)=y_{0}^{l_{k}}$. Q.E.D.

The first part of the following lemma follows from [8, Theorem (2.5)]; the second part, from Theorem (2.2.1) and the observation that $f^{*}$ takes $\operatorname{ker} f^{*}$ into $\operatorname{ker} \phi_{0}^{*}$.

Lemma (9.3.2). Let $z \in H^{*}(U)=H^{*}\left(U^{\prime}\right)$ be as in (9.3.1). Then z generates a sub-Hopf-algebra $(z)$ of $H^{*}\left(U^{\prime}\right)$, and $H^{*}\left(U^{\prime}\right)=(z) \otimes H^{*}\left(U^{\prime}\right) / /(z)$, as modules over $(z)$. Moreover, the Hopf-algebras $H^{*}\left(U^{\prime}\right) / /(z)$ and $\mathrm{im} f^{\prime *}$ are isomorphic.

Let us consider now the spectral sequence of the fibre map $f^{\prime}: G^{\prime} \rightarrow U^{\prime}$. By Proposition (5.2.1), the sheaf of coefficients $\mathcal{F}^{*}(K)$ over $U^{\prime}$ is simple, and $E_{2}\left(f^{\prime}\right)=H^{*}\left(U^{\prime}\right) \otimes H^{*}\left(K^{\prime}\right)$, as algebras. We claim

Lemma (9.3.3). $E_{2}\left(f^{\prime}\right)=\cdots=E_{s}\left(f^{\prime}\right)=H^{*}\left(U^{\prime}\right) \otimes H^{*}\left(K^{\prime}\right)$, where $s=l_{k}$, if $p=2$, and $2 l_{k}$, if $p>2$.

Proof. Observe first of all that the commutativity of the right portion of $C^{*}$ yields the fact that

$$
\operatorname{im} i^{*} \supset \operatorname{im} \phi_{k}^{*} .
$$

Now, by part (ii) of Theorem (2.2.1), $H^{*}\left(K^{\prime}\right)=\operatorname{im} \phi_{k}^{*} \otimes\{b\}$, where $\operatorname{deg} b$ $=s-1$. Hence every element of $H^{*}\left(K^{\prime}\right)$ whose degree is less than $(s-1)$ lies in im $\phi_{k}^{*}$. By $\left(^{*}\right)$ above, every such element of $H^{*}\left(K^{\prime}\right)$ lies in im $i^{\prime *}$. But the elements of im $i^{\prime *}$ are precisely those elements of $H^{*}\left(K^{\prime}\right)$ which are killed by all the differential operators $d_{r}$ for $r \geqq 2$. In particular, $d_{2}: H^{1}\left(K^{\prime}\right)=E_{2}^{0,1}\left(f^{\prime}\right)$ $\rightarrow E_{2}^{2,0}\left(f^{\prime}\right)$ is zero. By Proposition (5.4.3), $d_{2}=0$. Hence $E_{3}\left(f^{\prime}\right)=E_{2}\left(f^{\prime}\right)$. The rest of the proof follows by an easy induction. Q.E.D.

Lemma (9.3.4). Consider $E_{s}\left(f^{\prime}\right)=H^{*}\left(U^{\prime}\right) \otimes H^{*}\left(K^{\prime}\right)$. Then there is an element $b \in H^{s-1}\left(K^{\prime}\right)=E_{s}^{0,-1}\left(f^{\prime}\right)$ such that $d_{s}(b)=z \in H^{s}\left(U^{\prime}\right)=E_{s}^{s, 0}\left(f^{\prime}\right), z$ being the element defined in (9.3.1).

To prove it, just observe that $\phi_{0}^{*} \circ f^{*}(z)=f^{*}(z)=0$ because $f^{*}(z)=y^{l_{k}}$ $\in \operatorname{ch}\left(\phi_{g}\right) \subset \operatorname{ker} \phi_{0}^{*}$, by Theorem (2.2.1).

LemMa (9.3.5). $E_{s+1}\left(f^{\prime}\right)=\mathrm{im} f^{*} \otimes E(b, t-1) \otimes \mathrm{im} \phi_{k}^{*}$, as an algebra. Here $E(b, t-1)$ is an exterior algebra generated by an element $b \in E_{s+1}^{t-s, s-1}\left(f^{\prime}\right)$. 
Proof. By Lemma (9.3.2), we know that $H^{*}\left(U^{\prime}\right)=z \otimes H^{*}\left(U^{\prime}\right) / /(z)$ $=(z) \otimes \operatorname{im} f^{*}$, as modules over (z). Again by (9.3.4), $b$ is not in im $i^{*}$ (because $\left.d_{s}(b)=z \neq 0\right)$, and, hence, $b$ is not in im $\phi_{k}^{*}$. Thus, by Theorem (2.2.1), $H^{*}\left(K^{\prime}\right)$ $=\operatorname{im} \phi_{k}^{*} \otimes\{b\}$, as modules over im $\phi_{k}^{*}$. Therefore,

$$
E_{\imath}\left(f^{\prime}\right)=\left((z) \otimes i m f^{\prime *}\right) \otimes\left(\operatorname{im~} \phi_{k}^{*} \otimes(b)\right),
$$

and, hence

$$
E_{s+1}\left(f^{\prime}\right)=\left(i m f^{*}\right) \otimes\left(\operatorname{im~} \phi_{k}^{*}\right) \otimes E(\bar{b}, t-1),
$$

where $\bar{b}$ is represented by $b^{\prime}=z^{(t / s)-1} \otimes b \in E^{t-s, 8-1}\left(f^{\prime}\right)$. A simple computation shows that $b^{2}=0$. Q.E.D.

Observe that $d_{s+1}$ kills im $f^{\prime *} \otimes \operatorname{im} \phi_{k}^{*}$ since it kills each factor individually. Moreover, $d_{s+1}(b) \in E_{s+1}^{t+1,-1}=0$. Hence $d_{s+1}=0$ and, similarly, $d_{r}=0$, for all $r \geqq s+1$. Therefore,

Lemma (9.3.6). $E_{\infty}\left(f^{\prime}\right)=E_{\imath+1}\left(f^{\prime}\right)$.

Proposition (9.2) is a consequence of these lemmas.

\section{BiBLIOGRAPHY}

1. A. Borel, Sur l'homologie et la cohomologie des groupes de Lie compacts connexes, Amer. J. Math. 74 (1952), 273-342.

2. R. Bott and H. Samelson, Applications of the theory of Morse to symmetric spaces, Amer. J. Math. 80 (1958), 964-1029.

3. W. Browder, The cohomology of covering spaces of H-spaces, Bull. Amer. Math. Soc. 65 (1959), 140-141.

4. H. Cartan and S. Eilenberg, Homological algebra, Princeton Univ. Press, Princeton, N. J., 1956, pp. 254-256.

5. C. Chevalley and S. Eilenberg, Cohomology theory of Lie groups and Lie algebras, Trans. Amer. Math. Soc. 63 (1948), 85-124.

6. R. Godement, Topologie algebrique et theorie des faisceaux, Hermann, Paris, 1958.

7. J. Milnor, Construction of universal bundles. II, Ann. of Math. 63 (1956), 430-436.

8. J. Milnor and J. Moore, On the structure of Hopf-algebras, mimeographed notes, Princeton University, 1959.

9. D. Montgomery and L. Zippin, Topological transformation groups, Interscience, New York, 1955, pp. 188-189.

10. H. Samelson, Topology of Lie groups, Bull. Amer. Math. Soc. 58 (1952), 2-37.

11. A. Weil, L'integration dans les groupes topologiques et ses applications, Hermann, Paris, 1953, pp. 88-93.

Princeton University, Princeton, New Jersey

Massachusetts Institute of Technology, Cambridge, Massachusetts 